\title{
$1 \quad$ IL-1a secreted by subcapsular sinus macrophages promotes melanoma 2 metastasis in the sentinel lymph node by upregulating STAT3 signaling in 3 the tumor.
}

4 Tommaso Virgilio ${ }^{1,2}$, Joy Bordini ${ }^{1,3}$, Giulio Sartori $^{4}$, Irene Latino ${ }^{1}$, Daniel Molina-Romero ${ }^{1,5}$, Cristina

5 Leoni ${ }^{1}$, Murodzhon Akhmedov ${ }^{1,6}$, Andrea Rinaldi ${ }^{4}$, Alberto J. Arribas ${ }^{4}$, Diego Morone ${ }^{1}$, S. Morteza

6 Seyed Jafari ${ }^{7}$, Marina Bersudsky ${ }^{8}$, Aner Ottolenghi ${ }^{8}$, Ivo Kwee ${ }^{1,6}$, Anna Maria Chiaravalli ${ }^{9}$, Fausto

7 Sessa ${ }^{9}$, Robert E. Hunger ${ }^{7}$, Antonino Bruno ${ }^{10}$, Lorenzo Mortara ${ }^{11}$, Elena Voronov ${ }^{8}$, Silvia Monticelli ${ }^{1}$,

8 Ron N. Apte $^{8}$, Francesco Bertoni ${ }^{4,12}$, Santiago F. Gonzalez ${ }^{1^{*}}$

9 1: Institute for Research in Biomedicine, Università della Svizzera Italiana, Bellinzona, Switzerland.

10 2: Graduate School for Cellular and Biomedical Sciences, University of Bern, Bern, Switzerland.

11 3: Genomsys SA, Lugano, Switzerland.

12 4: Institute of Oncology Research, Università della Svizzera Italiana, Bellinzona, Switzerland.

13 5: Graduate School Ecole Polytechnique Fédérale de Lausanne, Lausanne, Switzerland.

14 6: BigOmics Analytics, Lugano, Switzerland.

15 7: Department of Dermatology, Inselspital, Bern University Hospital, University of Bern, Bern, Switzerland.

16 8: The Shraga Segal Department of Microbiology, Immunology and Genetics, Faculty of Health Sciences, Ben17 Gurion University of the Negev, Beer Sheva, Israel.

18 9: Unit of Pathology, ASST dei Sette Laghi, Department of Medicine and Surgery, University of Insubria, Varese, 19 Italy

20 10: Laboratory of Innate Immunity, Unit of Molecular Pathology, Biochemistry, and Immunology, IRCCS

21 MultiMedica, Milan, Italy.

22 11: Laboratory of Immunology and General Pathology, Department of Biotechnology and Life Sciences, University 23 of Insubria, Varese, Italy

24 12: Oncology Institute of Southern Switzerland (IOSI), Bellinzona, Switzerland

\section{Corresponding Author:}

35 Santiago Fernandez Gonzalez

36 Institute for Research in Biomedicine, via Vincenzo Vela 6.

$37 \mathrm{CH}-6500$ Bellinzona. Switzerland

38 Tel: (+41) 918200360

39 Email: santiago.gonzalez@irb.usi.ch 
ABSTRACT

41

42 During melanoma metastasization, tumor cells originated in the skin migrate via lymphatic vessels to the sentinel lymph node (sLN) in a process that facilitates their spread across the body. Here, we characterized the innate inflammatory responses to melanoma metastasis in the sLN. For this purpose, we confirmed the migration of fluorescent metastatic melanoma cells to the sLN and we characterized the inflammatory response in the metastatic microenvironment. We found that macrophages located in the subcapsular sinus (SSM), produce pro-tumoral IL-1 $\alpha$ after recognition of tumor antigens. Moreover, we confirmed that the administration of an anti-IL-1 $\alpha$ depleting antibody reduced metastasis. Conversely, the administration of recombinant IL-1 $\alpha$ accelerated the lymphatic spreading of the tumor. Additionally, the elimination of the macrophages significantly reduced the progression of the metastatic spread. To understand the mechanism of action of IL-1 $\alpha$ in the context of the lymph node microenvironment, we applied single-cell RNA sequencing to dissected metastases obtained from animals treated with an anti-IL-1 $\alpha$ blocking antibody. Amongst the different pathways affected, we identified STAT3 as one of the main targets of IL-1a signaling in metastatic cells. Moreover, we found that the anti-IL-1 $\alpha$ anti-tumoral effect was not mediated by lymphocytes, as IL-1R1 KO mice did not show any improvement in metastasis growth. Finally, we found a synergistic anti-metastatic effect of the combination of IL-1 $\alpha$ blocking and the STAT3 inhibitor (STAT3i) stattic. In summary, we described a new mechanism by which SSM support melanoma metastasis, highlighting a new target for immunotherapy.

KEYWORDS: Melanoma, Metastasis, Sentinel Lymph Node, IL-1a, STAT3,

\section{Macrophages, Subcapsular sinus, Immunotherapy, Inflammation.}




\section{INTRODUCTION}

Melanoma is the most lethal form of skin cancer and a serious threat for public health. In recent years, the incidence of this type of cancer has progressively increased and it is currently one of the most common malignancies in both adult and young individuals ${ }^{1}$. During melanoma development, malignant cells in the skin acquire genetic mutations that lead them towards the lymphatic vessels, which serve as a transportation system ${ }^{2}$. Once in the lymphatics, the metastatic cells initiate an active migration that leads them towards the sentinel $L N(s L N)^{3}$. The presence of melanoma metastasis in this organ is indicative of a poor prognosis, drastically decreasing the survival rate of the patients ${ }^{4,5}$.

Upon breaching the LN capsule, metastatic cells access the LN sinuses via the afferent lymphatics, following chemokine gradients generated by lymphatic endothelial cells ${ }^{6-8}$. The invasion of the SLN is initiated in the subcapsular sinus area (SS) $)^{6,8,9}$ and progressively spreads towards the inner structures of the $\operatorname{sLN}^{10}$. This process facilitates the access of the metastatic cells to the bloodstream via high-endothelial venules (HEVs) and their consequent spread to distant organs ${ }^{11-13}$.

The LN sinuses are populated by resident phagocytic cells, including three distinct macrophage subsets called subcapsular sinus macrophages (SSM), medullary macrophages (MM) and medullary cord macrophages (MCM), according to the area they reside ${ }^{14}$. Strategically positioned along the SS area, SSM are the first immune cells to encounter lymphtransported antigens and pathogens, preventing their systemic dissemination ${ }^{15}$. In addition, they play a critical role in the initiation of the immune responses against immune complexes and virus ${ }^{16-18}$ as well as promoting humoral immunity ${ }^{19,20}$. Despite the role of macrophages against infectious pathogens has been largely demonstrated, their involvement in the response against tumor remains somehow controversial ${ }^{21-27}$. This is mainly due to the ability of these cells to activate either anti- or pro-tumoral responses, according to their cell plasticity, that allows them being dicotomically classified in M1 and M2 macrophages ${ }^{28,29}$. For instance, some authors have described a protective function of SSM, which was associated with the capturing of dead tumor cell-derived antigens ${ }^{23}$ and their cross-presentation to CD8 ${ }^{+} \mathrm{T}$ cells ${ }^{21}$. Moreover, Tacconi and colleagues have recently suggested a protective role of CD169+ LN macrophages in breast cancer metastasis, which was dependent on the presence of $B$ cells ${ }^{27}$. Conversely, other studies have revealed a pro-tumoral effect of these cells, mainly linked with their capacity to trigger and maintain the inflammatory response both in peripheral and lymphoid tissues ${ }^{9,30,31}$. 
101 The inflammatory response plays a fundamental role in the behavior of cancer cells. Some 102 cancers, including melanoma, are able indeed to grow in chronically inflamed conditions and 103 to take advantage of inflammation ${ }^{32-35}$. One of the mechanisms by which innate inflammation 104 supports tumor growth is by the release of IL-1 family cytokines ${ }^{36,37}$. IL-1 $1 \beta$, the major 105 component of this family, has been shown to endorse different tumors, mainly by mediating 106 immune suppression and by activating endothelial cells ${ }^{38,39}$. Indeed, recent evidence suggests 107 that blocking the IL-1R signaling might prolong the survival time of patients with different 108 tumors ${ }^{40-44}$. In addition, IL-1 $\beta$ antagonism can synergize with immune checkpoint inhibitor 109 therapy ${ }^{38}$. However, the mechanisms responsible for this process might vary between the 110 primary tumor and the metastatic areas, including the $\mathrm{SLN}^{45,46}$. Understanding these 111 differences will influence the design of specific immunotherapies intended to control tumor 112 dissemination in both locations ${ }^{47,48}$ and in different types of tumors, including melanoma ${ }^{49,50 .}$

114 In the present work we characterized the innate immune response of the $\mathrm{SLN}$ to melanoma 115 metastasis invasion. Furthermore, we identified a novel mechanism that associates the 116 inflammatory reaction, initiated by SSM, to the progression of the metastatic melanoma cells. 117 These results will have a potential impact generating new therapies and improving the 118 efficiency of the current immunotherapies against metastatic melanoma, possibly acting on 119 macrophages, that represent the most abundant inflammatory cells infiltrating tumor. 
Development and characterization of a murine model of melanoma metastasis in the draining popliteal lymph node. To study the metastatic process in the sentinel lymph node (sLN) we transduced the melanoma cell line B16-F1 with a lentiviral vector codifying for mCherry and we characterized the expression of this fluorescent protein by FACS and microscopy (Fig. 1A and Supp. Fig. 1A, respectively). The primary tumor was induced by subcutaneous injection of the cancer cells in the mouse footpad, similarly to what was previously reported (Fig. 1B) ${ }^{51}$. Next, the formation of the primary tumor was monitored by measuring tumor volume (Supp. Fig. 1B,C) and tumor fluorescence was quantified by using the In Vivo Imaging System (IVIS; Fig. 1C,D). Following this approach, we observed a significant engraftment starting from week one post tumor implantation (p.t.i) (Fig. 1D). Subsequently, we identified macrometastases in the $S L N$ at three weeks p.t.i. (Fig. 1E). To study in more detail the progression of melanoma cells towards the $s L N$, we employed flow cytometry, observing a significant increase of the metastatic cells at day 15 p.t.i. compared to control samples (Fig 1F). In addition, to characterize the area of the metastasis, we used confocal microscopy analysis of the sLN at different time points following tumor induction (Fig. 1G). We measured a significant increase in the metastatic area starting at two weeks (Fig. $1 \mathrm{H})$. Besides, the morphology of SLN metastases showed that, in concordance with previous works in humans ${ }^{8}$, metastatic cells initially invade the subcapsular sinus area (SS) (Fig. 1G,I). Furthermore, at later time points, the metastasis progressively expanded through the interfollicular area (IF), invading the transverse sinus (Fig. 1I, Sup Fig. 1D). Conversely, we did not observe the presence of metastatic cells in distant organs, such as the spleen or the lungs, at equivalent time points (Fig. 1J), confirming the lymphatic dissemination of the tumor.

IL-1a promotes melanoma growth in the $s L N$. To characterize the inflammatory reaction induced by metastasis development, we quantified the total number of immune cells infiltrated in the sLN, observing a significant two-fold increase starting from the first week p.t.i. (Fig. 2A). More in detail, increases in dendritic cells (MHC- $-I^{+}, C D 11 c^{\text {high }}, \mathrm{CD} 11 \mathrm{~b}^{+}$and $\mathrm{CD} 11 \mathrm{~b}$ ), NK cells (CD3-, NK1.1 ${ }^{+}$), neutrophils (MHC-II-, GR $1^{\text {high }}$ ), monocytes (MHC-II-, GR $1^{\text {int }}$ ) and macrophages $\left(\mathrm{MHC}-\mathrm{II}^{+}, \mathrm{CD} 11 \mathrm{c}^{\text {intlow }}, \mathrm{CD} 11 \mathrm{~b}^{+}\right.$, Supp. Fig. 2A), as well as $\mathrm{B}\left(\mathrm{B} 220^{+}\right)$and T cells $\left(\mathrm{CD} 4^{+}, \mathrm{CD}^{+}\right.$, and $\mathrm{FOXP3}^{+} \mathrm{T}_{\text {reg}}$ ), were observed (Supp. Fig. 2B). To further characterize the recruitment of immune cells to the metastasized $S L N$, a multiplexed approach was applied to quantify the concentration of different inflammatory chemokines, including CXCL13, CXCL9, CCL22, CCL5 and CCL2, in the sLN supernatant, observing a significant increase at week three p.t.i. (Supp. Fig. 2C). Additionally, we measured the concentration of other 13 inflammatory 
p.t.i., compared to the control group. (Fig. 2B). A further study of the dynamics of IL-1 $\alpha$ release highlighted that the upregulation started at week two p.t.i. (Fig. 2C). To evaluate if IL-1a secretion was associated with other tumor models, including another solid tumor infiltrating the $s L N$, we measured the level of this cytokine in $S L N$ metastasized with melanoma B16-F10, or with the breast cancer cell line E0771, observing similar levels of IL-1 $\alpha$ in both models at three weeks p.t.i. (Fig. 2D).

Next, we hypothesized a pro-tumoral role of IL-1 $\alpha$ in the metastatic context. To explore this hypothesis, we treated mice with a daily subcutaneous injection of either IL-1a depleting

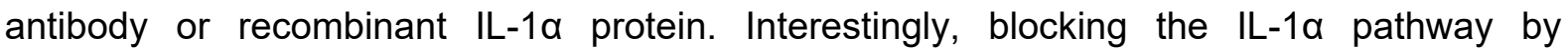
administration of the neutralizing antibody significantly decreased the metastasis growth in the $\mathrm{SLN}$, as indicated by a reduction in the number of metastatic cells measured by flow cytometric analysis at week three p.t.i.. Conversely, the number of melanoma cells significantly increased in the sLN treated with recombinant IL-1 $\alpha$ at equivalent time points (Fig. 2E). Moreover, the metastatic ratio, defined as the number of mice developing SLN metastasis at week three p.t.i. divided by the total number of mice showing primary tumor engraftment, was higher in the animals treated with recombinant IL-1 $\alpha$ and it was reduced following IL-1 $\alpha$ depletion (Fig. 2F). Nevertheless, the observed variation in the metastasis size after treatment could be dependent on the size of the primary tumor. Therefore, to discard that possibility, we normalized the number of metastatic cells to the primary tumor volume, confirming the results previously observed in Fig. 2E (Supp. Fig. 2D). Additionally, the previously described treatments did not have a significant effect on the growth of the primary tumor in comparison to the untreated control group (Supp. Fig. 2E). However, we observed that IL-1a KO mice showed a reduction not only of the metastatic cells at week three p.t.i. (Fig. 2G), but also of the primary tumor volume starting from the fourth week p.t.i. (Supp. Fig. 2F). This discrepancy could be partially explained by the mode of administration of the treatment, which promotes the transport towards the draining lymphatics, or by the time of administration of the compounds in comparison to the constant absence of IL-1 $\alpha$ in the tumor microenviroment in $\mathrm{KO}$ mice.

Subcapsular sinus macrophages are the main source of pro-tumoral IL-1a and disappear after tumor phagocytosis. In a previous study we characterized the role of LN macrophages as the main producers of IL-1 $\alpha$ in the $L N$, following influenza vaccination ${ }^{20}$. To elucidate the main source of IL-1 $\alpha$ in the melanoma model, we analyzed the infiltrating immune cells from the metastatic regions of the LN, by single cell RNA sequencing (scSeq, Fig. 3A).

190 Following this approach, we confirmed that LN macrophages are the main producers of IL-1 $\alpha$ 191 (Fig. 3 B, C). Moreover, the depletion of this population, following the injection with clodronate 
192 liposomes (CLL), significantly reduced the levels of IL-1 $\alpha$ in the LN supernatant (Fig 3D).

193 Importantly, depletion of macrophages following CLL administration completely abrogated the 194 growth of the metastatic melanoma cells in the LN, confirming their pro-tumoral nature (Supp.

195 Fig. 3A, B). However, the local administration of CLL did not affect the volume of the primary 196 tumor (Supp. Fig 3C). Next, to identify the specific subset of macrophages responsible for the 197 production of IL-1a, we used flow cytometry (Fig. 3E, F) and confocal microscopy (Fig. 3G), 198 which pointed out SSM (CD169 $\left.{ }^{+}, \mathrm{F} 4 / 80^{-}\right)$as the main source of IL-1a in the metastatic region. 199 To prove the relevance of these findings also in humans, we performed immunohistochemical 200 staining of melanoma metastatic LN from patients, confirming that the local production of IL201 1 $\alpha$ was associated with $\mathrm{CD}^{+} 8^{+}$tumor infiltrated macrophages in the SS region (Supp. Fig. 3D). 202 As suggested by other studies ${ }^{23}$, we also reported that tumor infiltrating SSM phagocyte melanoma cells (Fig. 3G, H, Supp. Fig. 3E, Supp. Mov. 1).

204 To investigate the mechanism of release of IL-1 $\alpha$ by SSM, we quantified cell numbers by flow 205 cytometry, observing that the total number of SSM remained constant during the first three weeks p.t.i. (Supp. Fig. 3F), while their frequency decreased (Supp. Fig. 3G). This was in contrast with a significant increase in the total number of macrophages observed at equivalent time points (Supp. Fig. 2A). Therefore, to investigate if SSM disappear following metastasis growth, we quantified by confocal microscopy the expression of the macrophage marker CD169 in different regions of the metastatic sLN. Interestingly, we observed that the CD169 layer in the SS was absent in the proximity of the metastatic area (Fig. 3I). More in detail, CD169 in the SS surrounding the metastatic region was expressed significantly less than in the non-metastasized SS (Fig. 3J), suggesting that SSM in direct contact with melanoma might undergo a cell death process. To confirm that the phagocytosis of tumor cell debris was able to induce SSM disappearance we injected B16-F1 lysate in the mouse footpad and performed flow cytometric analysis at 12 and $24 \mathrm{~h}$ following injection. We observed that the percentage of SSM significantly decreased compared with non-injected controls (Supp. Fig. 3H).

\section{SSM-derived IL-1a induces melanoma proliferation.}

In previous studies, we have demonstrated the involvement of $\mathrm{IL}-1 \alpha$ in the inflammatory reaction in the $\mathrm{LN}^{20,52}$. However, we did not observe here any significant effect in the recruitment of the major immune cells subtypes in the $s L N$ following treatment with anti-IL-1 $\alpha$ (Supp. Fig. 4 A, B). To further characterize the pro-tumoral mechanism of IL-1 $\alpha$, we measured the expression of IL-1R1, the only known receptor involved in the signaling of IL-1 $1 \alpha^{39}$, in the infiltrated cell types of a metastatic sLN. Amongst the evaluated cells, NK cells and melanoma displayed the highest level of IL-1R1 expression (Fig. 4A). To clarify the role of immune cells in mediating the pro-tumoral function of IL-1 $\alpha$, we induced melanoma in IL-1R1 KO mice, in 
which tumor microenvironment cells can't be involved in IL-1 signaling and only the tumor cells express this receptor. The absence of IL-1R1 in the immune compartment did not significantly affect neither the metastasis growth in the $s L N$ (Fig. 4B) nor the metastatic ratio (Fig. 4C), demonstrating that the pro-tumoral effect of IL-1 signaling was not associated with the immune cell response. Next, after confirming the expression of IL-1R1 in cultured melanoma cells (Supp. Fig. 4C), we measured their proliferation rate following exposure to IL-1a. We discovered that IL-1 $\alpha$ significantly promoted the proliferation of melanoma in murine and human cell lines (Fig. 4D and 4E, respectively). To further characterize the activation of IL1R1 signaling in B16-F1 melanoma cells, we measured by qPCR the expression of the gene codifying for the Myeloid Differentiation Primary Response 88 protein (MYD88), the main mediator of the Interleukin-1 receptor associated kinase (IRAK) signaling activated by IL$1 \mathrm{R} 1^{39}$. The results confirmed that melanoma cells treated with recombinant IL-1 $\alpha$ significantly upregulated the Myd88 gene (Fig. 4F).

IL-1a promotes aggressiveness of melanoma metastasis via STAT3. To study the pathways influenced by IL-1 $\alpha$ blocking in vivo, we performed scSeq of dissected metastases from mice treated with anti-IL-1 $\alpha$ antibody at three weeks p.t.i. (Supp. Fig. 5A). Next, we performed an influence analysis on the transcriptomic scSeq data (Fig. 5A) to identify the top ten IL-1 $\alpha$ key players, defined as the genes with the highest influence from all the il1a related pathways (Fig. 5B). Amongst them, we focused on STAT3, which was the most differentially expressed gene among the il1a key players following IL-1 $\alpha$ depletion (Fig. 5C). This gene codifies for the transcription factor STAT3, a well characterized mediator of aggressiveness in different cancers, including melanoma ${ }^{53,54}$. Furthermore, scSeq analysis of STAT3 expression highlighted the metastatic melanoma as the cells exhibiting the highest expression levels of this transcription factor (Fig. 5D). Moreover, the induction of the STAT3 gene in melanoma cells by IL- $1 \alpha$ was confirmed in vitro by the administration of recombinant IL-1 $\alpha$ (Fig. 5E). To recapitulate this mechanism at a functional level, we studied STAT3 protein expression and phosphorylation in murine melanoma cells using immunoblot assay. Treatment with recombinant IL-1 $\alpha$ induced a significant overexpression of STAT3 in comparison to untreated controls starting at $24 \mathrm{~h}$ post IL-1 $\alpha$ administration, while the addition of the anti-IL-1 $\alpha$ depleting antibody reverted this phenotype (Fig. 5F). Furthermore, exposure to recombinant IL-1 $\alpha$ induced the phosphorylation of STAT3, which was prevented by the depletion treatment (Fig. $5 \mathrm{G})$. To evaluate if this mechanism was also present in a human model, we quantified STAT3 and pSTAT3 in the A375 cell line post administration of human recombinant IL-1 $\alpha$, and we observed a significant increase of both total and phosphorylated forms in comparison to 
patients confirmed the expression and phosphorylation of STAT3 in the metastatic lesions (Supp. Fig. 5B, C). Once we confirmed the connection between IL-1 $\alpha$ and STAT3, we evaluated if a therapy with a STAT3 inhibitor (STAT3i) was able to improve the efficiency of the previously described IL-1a blocking therapy in the model of metastatic melanoma. Firstly, we observed that the administration of the combined therapies in vivo was able to contain the growth of the metastases more effectively than each of the two individual treatments (Fig. 5J). Additionally, we evaluated the combinatorial effect of these two therapies using an in vitro system, which highlighted a synergistic effect of the anti-IL-1 $\alpha$ blocking therapy combined with the STAT3i stattic (Fig. 5K, Supp. Fig. 5D, E). In more detail, the addition of stattic improved both the efficacy (Supp. Fig. 5F) and the potency (Supp. Fig. 5G, H) of the IL-1 $\alpha$ blocking

274 therapy.

275

\section{DISCUSSION}

In the present work, we characterized the innate immune responses of the sLN to melanoma metastasis invasion. We discovered that the SSM, which are the first immune cells to encounter melanoma metastasis in the sLN, phagocyted malignant cells and released IL-1 $\alpha$. Rather than triggering a tumor-killing inflammation, this cytokine increased metastatic cells aggressiveness by promoting STAT3 phosphorylation and increasing cancer proliferation. Importantly, blocking IL-1 $\alpha$ decreased metastatic growth and cooperated synergistically with STAT3 inhibition.

STAT3 is a transcriptional factor with a relevant role in melanoma progression ${ }^{55}$, together with relevant immunosuppressive and pro-angiogenic properties ${ }^{56,57}$. Diverse studies investigated the STAT3 pathway and its activation by IL-658. Of note, no studies reported similar effects of IL-1a. Based on this gap of knowledge, we stress a new target for metastatic melanoma therapy, acting on STAT3 signaling. This finding has further relevance in the context of combined therapies, which represent a very promising approach to target cancer cells at different levels, including tumor microenvironment ${ }^{59,60}$. In this context, blocking multiple immune pathways, such as IL-1 $\alpha$ and IL-6, might improve the efficacy of STAT3i in comparison to single or dual therapy, as suggested by other studies indicating the synergistic effect of these two cytokines ${ }^{36}$. In addition, considering the variability of cytokines levels and responses to cytokines-based therapies in patients ${ }^{61}$, IL-1a blockade could be envisaged as an alternative to IL- 6 inhibition for boosting STAT $3 \mathrm{i}^{62}$ in those patients with low levels of IL- 6 and low sensitivity to IL-6 depletion ${ }^{63}$. The specific cytokines expression profiling in patients, in fact, might be a useful tool to predict the patient response to the treatment and to design the best therapeutic strategy, according to the concept of personalized medicine, as previously 
proposed ${ }^{64-66}$. Moreover, IL-1 $\alpha$ blocking agents have already been tested in clinical trials on patients with various tumors and with different grading, showing variable efficiency $41,42,44,64$. Moreover, previous evidence, described a possible connection between PD-1 and IL-138,67 or STAT3 ${ }^{57,68}$ in various tumors, indicating that IL-1a blockade in combination with other therapies, including checkpoint inhibitors, might be object of further studies.

Another important aspect of the present work is the focus on metastasization. Metastases are in fact more dangerous than primary tumors for patients, especially in melanoma ${ }^{69}$. Moreover, their biology might differ substantially from the primary tumor ${ }^{70}$. For instance, STAT3 favors the spread of melanoma cells to distant organs, and it is particularly expressed in the melanoma metastasis ${ }^{53,54}$. For this reason, the identification of the IL-1 $\alpha$ - STAT3 axis, able to address efficiently metastases at their first stage, gains particular clinical relevance ${ }^{48,71}$.

311 Furthermore, the pro-tumoral effect of SSM over the metastatic melanoma might be associated with the more aggressive phenotype acquired by these cells in comparison to the primary tumor or to peripheral blood circulating melanoma cells ${ }^{11,12}$. However, other mechanisms, such as, for instance, the lymph-mediated protection from ferroptosis ${ }^{13}$, are also involved in this process.

317 In this study, we clearly characterized the pro-tumoral activity of SSM during melanoma 318 metastasis. However, previous evidence reported controversial functions of SSM in tumor biology 9,25,72. These cells, indeed, belong to the family of macrophages, which are capable of activating both pro- and anti- tumoral mechanisms, as a consequence of their cellular plasticity ${ }^{73}$. For this reason, targeting a specific pathway, as we proposed here, might reveal a better strategy than depleting the whole macrophage population, avoiding the hampering of possible anti-metastatic functions of these cells ${ }^{23,24,74}$. Similarly, the development of drugs capable of targeting specifically SSM might reveal useful to block the IL-1 $\alpha$ - STAT3 axis only in these cells and to boost their anti-tumoral properties, in a process of macrophages repolarization ${ }^{75}$. Unfortunately, despite recent studies described compounds capable of localizing differentially in the SCS and in the medullary area of the LN, a therapy able to differentiate specifically macrophage subsets in the $L N$ is still missing $72,76,77$.

Additionally, SSM initiate the inflammatory response in the $S L N$ by different mechanisms, including cell death associated with the release of pre-stored IL-1 $\alpha^{20,78}$, which functions as an alarmin molecule following release from dying cells ${ }^{79}$. However, despite we observed a prominent recruitment of immune cells, we have not detected an efficient tumor-killing. 
336 cells, or a specific inhibition of IL-1R1 by IL-1R antagonist in the immune compartment, which 337 has been previously suggested in a study in human patients ${ }^{80}$. These and other hypotheses 338 will furnish exciting insights on novel methods to improve immunotherapy and should be 339 investigated in the future.

341 In conclusion, our results provide evidence of a novel function of SSM in melanoma metastasis

342 progression by controlling the IL-1 $\alpha$ - STAT3 axis. Importantly, IL-1 $\alpha$ blocking decreased 343 metastasis growth and acted synergistically with a STAT3i in controlling tumor growth. Taken 344 together, these findings provide with new opportunities to improve currently available 345 immunotherapies against metastatic melanoma. 


\section{Cell culture and lentiviral transduction}

348 B16-F1, B16-F10 and A375 cell lines were provided, respectively, by G. Guarda (IRB, 349 Bellinzona) and C. Catapano (IOR, Bellinzona). E0771 cell lines were acquired from Ch3 350 BioSystems. All cell lines were maintained in a complete RPMI medium (RPMI+Hepes, $10 \%$ heat inactivated FBS, 1 \% Glutamax, 1 \% sodium pyruvate, $1 \%$ non-essential amino acids, 50 units $/ \mathrm{mL}$ Penicillin, $50 \mu \mathrm{g} / \mathrm{mL}$ Streptomycin and $50 \mu \mathrm{M} \beta$-mercaptoethanol) and regularly tested for mycoplasma (MycoAlert Mycoplasma Detection kit, Lonza). The B16-F1-mCherry and B16-F1-Azurite cell lines were generated by lentiviral transduction. Briefly, lentiviral plasmids pSicoR-Ef1a-mCh (Addgene 31847) or pLV-Azurite (Addgene 36086) were transfected in HEK293T cells with packaging vectors pMD2G and psPAX (Addgene 12260 and 12259) to generate viral particles. After concentration by centrifugation, the virus was later collected and used for B16-F1 transduction. Transduced fluorescent cells were selected by live cells sorting using BD FACSAria Sorter.

\section{Mice}

361 The Institute for Research in Biomedicine (IRB) hosted animal experiments in facilities defined 362 as specific pathogen-free facilities, according to FELASA guidelines. Experiments involving 363 IL-1 $\mathrm{KO}$ mice were conducted at the Ben Gurion University animal facility. In both facilities mice were housed in Individually Ventilated Cages (IVC) with controlled light : dark cycle (12 : 12), room temperature $\left(20-24{ }^{\circ} \mathrm{C}\right)$ and relative humidity $(30-70 \%)$. Animal caretakers, researchers and veterinarians provided mice with daily check of general health conditions. All animal experiments have been conducted in accordance with the Swiss Federal Veterinary Service guidelines and the Israel Animal Welfare Act. All mouse procedures have been previously authorized by the relevant institutional committee (Commissione Cantonale per gli Esperimenti sugli Animali) of the Cantonal Veterinary Office and by the by the Israeli Council for Animal Experimentation of the Ministry of Health, with licensing numbers TI 25/2017, TI 24/2018, TI 55/2018 and TI 30/2020. Charles River Laboratories, F. Sallusto (IRB, Bellinzona) and R. Apte (BGU, Be'er Sheva) provided, respectively, C57BL/6J, B6.129S7-II1r1tm1/mx/J

374 (IL-1R1KO/KO, Jackson code 003245) and IL-1 $\alpha$ KO mice ${ }^{81}$, which were then bred in-house.

375 B6.129P2(Cg)-Cx3cr1tm1Litt/J (CX3CR1GFP/wt) mice were originally acquired from Jackson Laboratories (cat 005582) and bred in-house. The genotype of all mice was confirmed as previously described ${ }^{82,83}$. Mice in an age range of $6-12$ weeks, showing good health conditions and no abnormal clinical signs took part in the experiments. Equal numbers of males and females were assigned to experimental groups through a statistical randomization

380 process. Power calculation per groups size determination, performed by using $\mathrm{R}$ software (R: 381 A Language and Environment for Statistical Computing, R Core Team, R Foundation for 
382 Statistical Computing, Vienna, Austria), estimated a number of 10 animals per group to obtain $383>99 \%$ statistical power.

\section{Allograft model}

$10^{6}$ cells from the syngeneic cell lines B16-F1, B16-F10 and E0771 were injected subcutaneously in the right footpad in $10 \mu \mathrm{L}$ sterile PBS. Mice were anesthetized with Isoflurane (5 \% for induction, $3 \%$ for maintenance, $\mathrm{FiO} 2=1,1 \mathrm{~L} / \mathrm{min}$ ) and monitored, after cells injection and anesthesia recovery, to check for absence of pain or impaired movement. Mouse body weight and tumor size were measured every one or two days. Tumor volume was calculated with the formula $V=\left(\right.$ length $\mathrm{x}$ width $\left.{ }^{2}\right) / 2$ and mice were euthanized when tumor reached $250 \mathrm{~mm}^{3}$. Euthanasia was performed by isoflurane overdose followed by cervical dislocation and immediate organs collection. We excluded from experiments mice which did not develop tumor ( $\mathrm{V}=0 \mathrm{~mm}^{3}$ at day 20 p.t.i. $)$ or which developed tumor in the popliteal fossa, impeding the collection of the popliteal lymph node. In some experiments, we injected $15 \mu \mathrm{L}$ of the B16-F1 tumor cell lysate originated from $5 \times 10^{5}$ cells, sonicated at constant cycles of 30 seconds.

\section{In vivo treatments}

398 To maximize the specific effect of treatments on LN metastases and to minimize the effect on tumor engraftment and primary tumor growth, all treatments were administered when the primary tumor reached a size of $40 \mathrm{~mm}^{3}$, which corresponds to the time of arrival of the first metastatic cells to the LN. Additionally, all local treatments were injected in the calf, to minimize their distribution to the primary tumor. All treatments were resuspended in a maximum volume of $10 \mu \mathrm{L}$ in calcium- and magnesium-free PBS (PBS-). After injection in the subcutis, mice were recovered from anesthesia and monitored for absence of any sign of pain in the foot. Carrier-free recombinant mouse IL-1 $\alpha$ (Biolegend) was locally administered at a dose of $1 \mu \mathrm{g}$ / $10 \mu \mathrm{L}$ per day. The anti-mouse IL-1 $\alpha$ monoclonal antibody (InVivoMAb anti-mouse IL-1 $\alpha$, clone ALF-161, BioXCell) was administered to deplete IL-1 $\alpha$ at a dose of $200 \mu \mathrm{g}$ i.v. plus 60 $\mu$ locally, as previously reported ${ }^{20}$. Depletion was then maintained with a daily local injection of $60 \mu \mathrm{g}$. STAT3 was inhibited by local injection of stattic (SelleckChem) $3.75 \mathrm{mg} / \mathrm{kg}$ every two days. Stattic was reconstituted, according to manufacturer's instructions, in $5 \%$ DMSO

411 (VWR), 40 \% PEG300 (MedChem Express), $5 \%$ Tween® 80 (Sigma-Aldrich) and $50 \%$ 412 distilled water. For macrophages depletion, mice received locally $10 \mu \mathrm{L}$ of clodronate- or PBScontaining liposomes (Liposoma), followed by a second dose two days later.

\section{IVIS}

415 To monitor primary tumor growth and mCherry expression of fluorescent cancer cells, we used 416 the IVIS Spectrum Imaging System (Caliper LifeSciences). Mice were anesthetized with 417 isoflurane as above described to measure epifluorescence. Immediately after image 
418 acquisition, animals were recovered from anesthesia. Images were later analyzed using Living

419 Image Software 4.2 (Caliper LifeSciences).

\section{Immunofluorescence and immunohistochemistry}

421 For mouse microscopy experiments, organs were fixed immediately after collection in $4 \%$ 422 paraformaldehyde (Merck-Millipore) for $12 \mathrm{~h}$ at $4{ }^{\circ} \mathrm{C}$, then washed in calcium- and magnesium423 free PBS (PBS-) and embedded in 4 \% Low Gelling Temperature Agarose (Sigma-Aldrich). $42450 \mu \mathrm{m}$ sections were cut with a vibratome (VT1200S, Leica). Slices were stained in a blocking 425 buffer composed of TritonX100 (VWR) 0.1-0.3 \%, BSA 5\% (VWR) and fluorescently labelled 426 antibodies at proper concentration, all diluted in PBS supplemented with calcium and 427 magnesium (PBS+). After 72 hours of incubation at $4{ }^{\circ} \mathrm{C}$, samples were washed in $0.05 \%$ 428 Tween® 20 (Sigma-Aldrich), post-staining fixed with PFA $4 \%$, washed in PBS- and mounted 429 on glass slides. Confocal images were acquired using a Leica TCS SP 5 microscope with a 20 $430 \times 0.7$ oil objective. To quantify the rate of invasion of melanoma in each sLN region, we first 431 identified metastatic mass on the mCherry channel with an automatic Otsu threshold, after 432 noise filtering with ImageJ Despeckle plugin and size filtering for regions bigger than $30 \mu \mathrm{m}^{2}$. 433 LN regions were manually identified based on CX3CR1 and CD21/35 morphology. Next, we 434 quantified the total tumor area and the percentage of overlap of metastasis with each other 435 LN region, respectively. Sample sizes were distributed as follows: $n=21,7$ and 11 for week 436 one, two and three p.t.i., respectively. To quantify the expression of CD169+, the $\mathrm{LN}$ regions 437 were manually identified as described above and the mean fluorescence intensity of each 438 region was later calculated. To stain IL-1 $\alpha$ in human lymph nodes, samples were stained using 439 the BOND-III fully automated IHC/ISH stainer (Leica Biosystems) according to the 440 manufacturer's instructions. To stain STAT3 and pSTAT3, primary antibodies (mouse anti441 Stat3, clone 124H6, and mouse anti-Phospho-Stat3, Tyr705, clone M9C6, Cell Signaling) 442 were incubated overnight at $4^{\circ} \mathrm{C}$ and the MACH4 Universal HRP-Polymer Detection System 443 (Biocare Medical) was applied according to the manufacturer's protocol. 3D cell reconstruction 444 was performed using Imaris Cell Imaging Software (Oxford Instruments).

\section{Flow Cytometry}

446 LNs were collected, disrupted with tweezers, and enzymatically digested for 10 minutes at 37 $447{ }^{\circ} \mathrm{C}$. DNase I $(0.28 \mathrm{mg} / \mathrm{mL}$, VWR $)$, dispase $(1 \mathrm{U} / \mathrm{mL}$, Corning) and collagenase $\mathrm{P}(0.5 \mathrm{mg} / \mathrm{ml}$, 448 Roche) were resuspended in calcium- and magnesium-free PBS (PBS-). Digestion was 449 stopped using a solution of 2 mM EDTA (Sigma-Aldrich) and $2 \%$ heat-inactivated filter450 sterilized fetal calf serum (Thermo Fisher Scientific) diluted in PBS- (Sigma-Aldrich). Fc 451 receptors were blocked ( $\alpha C D 16 / 32$, BioLegend) followed by surface staining and analyzed by 452 flow cytometry on an LSRFortessa or FACSymphony (BD Biosciences). For IL-1a detection, 453 intracellular staining was performed with a dedicated kit (88/8824/00, eBioscience), following 
the manufacturer's instructions. Data were analyzed using FlowJo software (FlowJo LLC). To measure cytokines and chemokines expression in the LN, LEGENDPlex assays (Mouse Proinflammatory Chemokine Panel and Mouse Inflammation Panel; BioLegend) were used. Briefly, pLNs were collected and carefully disrupted in $75 \mu \mathrm{L}$ ice-cold phosphate buffer, minimizing cell rupture. The suspension was centrifuged at 100 rcf for 5 minutes and the supernatant was collected. $25 \mu \mathrm{L}$ supernatant was used for cytokines and chemokines detection. Samples were analyzed by flow cytometry on an LSRFortessa or FACSymphony (BD Biosciences) and data were analyzed using LEGENDPlex software (BioLegend).

\section{Antibodies}

463 The list of antibodies used to stain mouse samples includes anti-CD21/35 (CR1/CR2, clone 464 7E9, BioLegend), anti-podoplanin (clone eBio8.1.1, Invitrogen), anti-CD3 (clone 17A2, 465 BioLegend), anti-B220 (CD45R, clone RA3-6B2, BioLegend), anti-Gr-1 (clone RB6-8C5, 466 BioLegend), anti-NK1.1 (clone PK136, BioLegend), anti-MHC II (I-A/I-E, clone M5/114.15.2, 467 BioLegend), anti-CD11b (clone M1/70, BioLegend), anti-CD11c (clone N418, BioLegend), 468 anti-F4/80 (clone BM8, BioLegend), anti-CD169 (Siglec-1, clone 3D6.112), anti-IL-1R1 (clone 469 FAB7712N, R\&D Systems), anti-IL-1a (clone ALF-161, BioLegend; clone REA288, Miltenyi 470 Biotec), anti-CD4 (clone RM4-5, BioLegend), anti-CD8a (clone 53-6.7, Invitrogen), anti-CD25 471 (clone PC61, BioLegend). Human samples were stained with anti-IL-1 $\alpha$ (clone OTI2F8, Novus 472 Biologicals), and anti-CD68 antibodies (clone PG-M1, Dako).

\section{Single-cell RNA-sequencing}

474 Metastatic LN were obtained from four PBS injected mice, six tumor-bearing mice and four 475 tumor-bearing mice treated with anti-IL-1 $\alpha$ as described above. Metastases from tumor476 bearing mice were microsurgically dissected using sterile micro-surgical tools. SS, IF and F 477 regions were dissected in negative controls. Later, samples were disrupted into single cell 478 suspension as described for flow cytometry, using sterile nuclease-free tools. Single cells were 479 barcoded using the 10x Chromium Single Cell platform, and cDNA libraries were prepared 480 according to the manufacturer's protocol (Single Cell 3' v3, 10x Genomics, USA). In brief, cell 481 suspensions, reverse transcription master mix and partitioning oil were loaded on a single cell 482 chip, then run on the Chromium Controller. Reverse Transcription was performed within the 483 droplets at $53{ }^{\circ} \mathrm{C}$ for 45 minutes. cDNA was amplified for a 12 cycles total on a Biometra 484 thermocycler. cDNA size selection was performed using SpriSelect beads (Beckman Coulter, 485 USA) and a ratio of SpriSelect reagent volume to sample volume of 0.6. cDNA was analyzed 486 on an Agilent Bioanalyzer High Sensitivity DNA chip for qualitative control purposes. cDNA 487 was fragmented using the proprietary fragmentation enzyme blend for 5 minutes at $32{ }^{\circ} \mathrm{C}$, 488 followed by end repair and A-tailing at $65{ }^{\circ} \mathrm{C}$ for 30 minutes. cDNA was double-sided size 489 selected using SpriSelect beads. Sequencing adaptors were ligated to the cDNA at $20{ }^{\circ} \mathrm{C}$ for 
15 minutes. cDNA was amplified using a sample-specific index oligo as a primer, followed by another round of double-sided size selection using SpriSelect beads. Final libraries were analyzed on an Agilent Bioanalyzer High Sensitivity DNA chip for quality control. cDNA libraries were sequenced on a NextSeq500 Illumina platform aiming for 50,000 reads per cell. Base calls were converted to reads with the software Cell Ranger (10x Genomics; version 3.1) Quality control, processing, annotation, and differential gene expression analysis of single-cell RNA-sequencing data

We used the cellranger pipeline ${ }^{84}$ to generate gene expression count matrices from the raw data. For each sample, a gene-by-cell counts matrix was used to create a Seurat object using Seurat ${ }^{85,86}$. We filtered cell barcodes with $<500$ UMls and $>5 \%$ mitochondrial contents. Each individual sample was then normalized by a factor of 10,000 and log transformed (NormalizeData). The top 2000 most variable genes were then identified within each sample using the FindVariableFeatures method. We then integrated the cells from all samples together using FindIntegrationAnchors and IntegrateData (2000 genes). The integrated gene expression matrix obtained by applying the filtering steps above was then used to perform principal component analysis (RunPCA), preliminary clustering analysis, including nearest neighbour graph (FindNeighbors) and unbiased clustering (FindClusters), and cell type annotation. Uniform Manifold Approximation and Projection (UMAP) was then used to visualise the integrated expression data. We identified gene expression markers for each cluster using FindAllMarkers from Seurat with default settings, including Wilcoxon test and Bonferroni $p$ value correction ${ }^{85,86}$. Differential gene expression between specified clusters (or

511 subclusters) was performed using FindMarkers (Wilcoxon rank sum test) with Benjamini512 Hochberg false discovery rate (FDR) correction, average log fold change (logFC) and 513 detection/expression percentage rate (pct). Genes were considered (significantly) differentially 514 expressed if FDR $<0.05$, logFC $>0.2$ and $p c t>20 \%$ within the cells in a given group.

\section{Gene relevance analysis of single-cell RNA sequencing data}

516 To determine gene relevance across the single cell RNA sequencing data, we used a network 517 science approach. To study nodes relevance we applied Graph Theory rules ${ }^{87,88}$ using 518 mathematical and social network analysis concepts. We restricted the analysis to protein519 protein interactions and to the pathways in which the gene il1a is involved. Relevant pathways 520 (Cytokine-cytokine receptor interaction, Necroptosis, Hematopoietic cell lineage, Type I 521 diabetes mellitus, Pertussis, Leishmaniasis, Tuberculosis and Inflammatory bowel disease) 522 and related were selected using the Kyoto Encyclopedia of Genes and Genomes ${ }^{89}$. Then we 523 measured the expression of these genes in our scRNA-Seq dataset and we used their 524 expression values as input of STRING ${ }^{90}$. The resulting graph was used for the network 525 analysis. We implemented a previously described comprehensive algorithm for evaluating 
526 node influences in social networks ${ }^{91}$. This algorithm is based on three centrality measures:

527 eigenvector centrality ${ }^{92}$, current flow betweenness centrality ${ }^{93,94}$ and reachability ${ }^{95}$.

528 Eigenvector centrality computes the centrality for a node based on the centrality of its

529 neighbors. Current-flow betweenness centrality starts from an electrical current model

530 describing the spreading pattern, to which betweenness centrality, which uses shortest paths,

531 is applied. Finally, reachability refers to the local reaching centrality of a node in a directed

532 graph as the proportion of other nodes reachable from that node. In addition single cell CMP

533 values are taken as weights of the nodes. Basing on these parameters, the algorithm ranked

534 node influences by analyzing preference relations and performing random walk. In the first

535 step a partial preference graph (PPG) is derived from the analysis of preference relation

536 between every node pair for each measure. Later, the comprehensive preference graph (CPG)

537 originated from the combination of preference relations and the three previously indicated

538 measures. Finally, a random walk was executed on CPG to determine node effect. By applying

539 this implementation to scSeq data, it was possible to obtain a list of genes related to il1a

540 pathways, according to their importance in our dataset.

541 Proliferation (MTT)

542 To evaluate tumor cells proliferation and response to treatments, B16-F1 and A375 cells were

543 seeded in a 96-well plate. Carrier-free recombinant mouse (Biolegend) or human

544 (SinoBiological) IL-1 $\alpha$ were administered at $10 \mathrm{ng} / \mathrm{mL}$ and cells were incubated, respectively,

545 for 24 hours or 72 hours. Anti-mouse IL-1 $\alpha$ monoclonal antibody (InVivoMAb anti-mouse IL-

546 1a, clone ALF-161, BioXCell) was administered at the indicated doses 24 hours before data

547 collection. To inhibit STAT3, stattic (SelleckChem) was administered at the indicated dosages.

548 Proliferation was assessed by MTT (Methylthiazolyldiphenyl-tetrazolium bromide) assay

549 according to the manufacturer's recommendations (Sigma). Absorbance (OD, $560 \mathrm{~nm}$ ) was

550 measured in a microplate reader (Cytation 5, BioTek). Sensitivity to single drug treatments

551 was evaluated by IC50 (4-parameters calculation upon log-scaled doses), as previously

552 reported ${ }^{96}$. The beneficial effect of the combinations versus the single agents was considered

553 both as synergism according to the Chou-Talalay combination index ${ }^{97}$, as previously

554 performed ${ }^{96,98}$, and as potency and efficacy according to the MuSyC algorithm ${ }^{99}$.

555 qPCR

556 To measure the expression of STAT3, Myd88 and Gapdh genes, the following sets of primers 557 were designed: STAT3 forward, 5'-CACAAATATTTTTGAGTCGGCGC-3'; STAT3 reverse 5'558 AAAGCCCCCGATGAGGTAATTC-3'; Myd88 forward, 5'-CGGCAACTAGAACAGACAGACT-

559 3'; Myd88 reverse, 5'-GCAAACTTGGTCTGGAAGTCAC-3'; Gapdh forward, 5'560 ACATCATCCCTGCATCCACT-3' ; Gapdh reverse, 5'-AGATCCACGACGGACACATT-3'. To 561 isolate RNA from cell culture, cells were disposed of in single-cell suspension in calcium- and 
562 magnesium-free PBS (PBS-). RNA was isolated using an RNAeasy Mini kit (QIAGEN). Two

$563 \mu \mathrm{g}$ of cDNA were synthesized using a cDNA synthesis kit (Applied Biosystems) following the

564 manufacturer's recommendations. For the qPCR reaction, a SYBR Master Mix (Applied

565 Biosystems) was used, and samples were run on a QuantStudioTM 3 Real-Time PCR System

566 (Thermofisher). mRNA levels were expressed relative to GAPDH expression. The Pfaffl

567 method ${ }^{100}$ was used to calculate the relative expression of the transcripts.

\section{Immunoblotting}

569 To evaluate protein expression in tumor cells, B16-F1 and A375 cells were treated using 570 carrier-free recombinant mouse (Biolegend) or human (SinoBiological) IL-1 $\mathrm{\alpha}$, at $100 \mathrm{ng} / \mathrm{mL}$.

571 To block IL-1 $\alpha$, anti-mouse IL-1 $\alpha$ monoclonal antibody (InVivoMAb anti-mouse IL-1 $\alpha$, clone

572 ALF-161, BioXCell) was administered at the dose of $100 \mathrm{ng} / \mathrm{mL}$. All treatments were

573 administered either for 24 hours or 72 hours. Cells were harvested and lysed by boiling

574 samples in 2x Laemmli sample buffer (BioRad), supplemented with $\beta$-mercaptoethanol

575 (Merck), for 10 minutes. Lysates (30-50 $\mu \mathrm{g})$ were resolved according to molecular weight by

576 electrophoresis using Mini-PROTEAN TGX Precast gels 4-20\% gradient (BioRad). Next,

577 proteins were blotted onto nitrocellulose membrane (BioRad) by electric transfer and the

578 membranes were blocked in TBST (20 mM Tris-HCl [pH 7.5], $150 \mathrm{mM} \mathrm{NaCl}, 0.1$ \% Tween 20)

579 with $5 \%$ nonfat dry milk (BioRad) for one hour at room temperature. The following primary

580 antibodies were used in TBST $5 \%$ BSA buffer: mouse monoclonal, anti-Stat3 (clone 124H6,

581 9139, Cell Signaling Technology) and rabbit monoclonal, anti-p(Y705)Stat3 (9131, Cell

582 Signaling Technology). Mouse monoclonal anti-GAPDH (clone FF26A/F9, CNIO) was used in

583 TBST with $5 \%$ nonfat dry milk. The secondary antibodies used were: ECL anti-mouse IgG

584 horseradish peroxidase-linked species-specific whole antibody and ECL anti-rabbit IgG

585 horseradish peroxidase-linked species-specific whole antibody (GE Healthcare). Membranes

586 were treated with Westar $\eta \mathrm{C} 2.0$ chemiluminescent substrate (Cyanagen) and signals were

587 detected using digital imaging with Fusion Solo (Vilber Lourmat).

\section{Statistical analyses}

589 All raw data were analyzed, processed and presented using GraphPad Prism 8.2.1 (Graphpad

590 Software, La Jolla, USA). First, we applied the Shapiro-Wilk normality test to analyze the 591 distribution of data. Then we compared means among groups using One-Way ANOVA or 592 Unpaired t test for data with normal distribution, and the non-parametric Kruskal-Wallis or 593 Mann-Whitney test for groups which did not present a normal distribution. In all statistical tests $594 P$ value is indicated as * when $<0.05$, ${ }^{* *}$ when $<0.005$, ${ }^{* * *}$ when $<0.0005$, ${ }^{* * * *}$ when $<0.0001$. 


\section{ACKNOWLEDGMENTS}

596 We thank Federica Sallusto (IRB, Bellinzona, Switzerland), Carlo Catapano (IOR, Bellinzona,

597 Switzerland) and Greta Guarda (IRB, Bellinzona, Switzerland) for providing, respectively, IL598 1R1 KO mice, A375, B16-F1 and B16-F10 cell lines. We also thank David Jarossay (IRB, 599 Bellinzona, Switzerland) for assistance in flow cytometry and live cell sorting experiments, 600 Diego Ulisse Pizzagalli (IRB, Bellinzona, Switzerland) for the support in Imaris Software 601 usage, Kevin Ceni (IRB, Bellinzona, Switzerland) for Supp. Mov. 1 editing, and Rocco 602 D'Antuono (IRB, Bellinzona, Switzerland, now Francis Crick Institute, London, United 603 Kingdom) for microscopy support. 


\section{REFERENCES}

605

606 1. Carr S, Smith C, Wernberg J. Epidemiology and Risk Factors of Melanoma. Surg Clin

607 North Am. 2020;100(1):1-12. doi:10.1016/j.suc.2019.09.005

608

2. Karlsson MC, Gonzalez SF, Welin J, Fuxe J. Epithelial-mesenchymal transition in

609

610

611

3. Damsky WE, Rosenbaum LE, Bosenberg M. Decoding melanoma metastasis.

612

613

4. Ulmer A, Dietz K, Hodak I, et al. Quantitative Measurement of Melanoma Spread in

614

615 cancer metastasis through the lymphatic system. Mol Oncol. 2017;11(7):781-791. doi:10.1002/1878-0261.12092

616

5. JE G, RA S, KR H, et al. Melanoma staging: Evidence-based changes in the

617

618 American Joint Committee on Cancer eighth edition cancer staging manual. $C A$ Cancer J Clin. 2017;67(6):472-492. doi:10.3322/CAAC.21409

619

6. Wiley HE, Gonzalez EB, Maki W, Wu MT, Hwang ST. Expression of CC chemokine

620

621 receptor-7 and regional lymph node metastasis of B16 murine melanoma. $J$ Natl

622 Cancer Inst. 2001;93(21):1638-1643. doi:10.1093/jnci/93.21.1638

623

7. A B-B. Organ selectivity in metastasis: regulation by chemokines and their receptors. Clin Exp Metastasis. 2008;25(4):345-356. doi:10.1007/S10585-007-9097-3

625

626

8. Das S, Sarrou E, Podgrabinska S, et al. Tumor cell entry into the lymph node is controlled by CCL1 chemokine expressed by lymph node lymphatic sinuses. J Exp

627 Med. 2013;210(8):1509-1528. doi:10.1084/jem.20111627

628

9. Singh R, Choi BK. Siglec1-expressing subcapsular sinus macrophages provide soil

629 for melanoma lymph node metastasis. Elife. 2019;8. doi:10.7554/eLife.48916

630

10. Kretschmer L, Mitteldorf C, Hellriegel S, et al. The sentinel node invasion level (SNIL) as a prognostic parameter in melanoma. Mod Pathol 2021. Published online June 15, 2021:1-11. doi:10.1038/s41379-021-00835-5

633

11. Brown M, Assen FP, Leithner A, et al. Lymph node blood vessels provide exit routes for metastatic tumor cell dissemination in mice. Science (80- ). 2018;359(6382):14081411. doi:10.1126/science.aal3662 
638

639

640

641

642

643

644

645

646

647

648

649

650

651

652

653

654

655

656

657

658

659

660

661

662

663

664

665

666

667

668

669

670

671

672

13. Ubellacker JM, Tasdogan A, Ramesh V, et al. Lymph protects metastasizing melanoma cells from ferroptosis. Nature. 2020;585(7823):113-118.

doi:10.1038/s41586-020-2623-z

14. Gray EE, Cyster JG. Lymph node macrophages. J Innate Immun. 2012;4(5-6):424436. doi: $10.1159 / 000337007$

15. Kuka M, lannacone $M$. The role of lymph node sinus macrophages in host defense. Ann N Y Acad Sci. 2014;1319(1):38-46. doi:10.1111/NYAS.12387

16. YR C, FD B. B cells acquire particulate antigen in a macrophage-rich area the boundary between the follicle and the subcapsular sinus of the lymph node. Immunity. 2007;27(1):160-171. doi:10.1016/J.IMMUNI.2007.06.007

17. M I, EA M, E T, et al. Subcapsular sinus macrophages prevent CNS invasion on peripheral infection with a neurotropic virus. Nature. 2010;465(7301):1079-1083. doi:10.1038/NATURE09118

18. SF G, V L-K, MP K, et al. Capture of influenza by medullary dendritic cells via SIGN$\mathrm{R} 1$ is essential for humoral immunity in draining lymph nodes. Nat Immunol. 2010;11(5):427-434. doi:10.1038/NI.1856

19. M G, A C, B M, et al. Host response. Inflammation-induced disruption of SCS macrophages impairs $B$ cell responses to secondary infection. Science. 2015;347(6222):667-672. doi:10.1126/SCIENCE.AAA1300

20. Chatziandreou N, Farsakoglu Y, Palomino-Segura M, et al. Macrophage Death following Influenza Vaccination Initiates the Inflammatory Response that Promotes Dendritic Cell Function in the Draining Lymph Node. Cell Rep. 2017;18(10):24272440. doi:10.1016/j.celrep.2017.02.026

21. Asano K, Nabeyama A, Miyake Y, et al. CD169-Positive Macrophages Dominate Antitumor Immunity by Crosspresenting Dead Cell-Associated Antigens. Immunity. 2011;34(1):85-95. doi:10.1016/j.immuni.2010.12.011

22. Kumamoto K, Tasaki T, Ohnishi K, et al. CD169 Expression on Lymph Node Macrophages Predicts in Patients With Gastric Cancer. Front Oncol. 2021;11:824. doi:10.3389/FONC.2021.636751

23. Moalli F, Proulx ST, Schwendener R, Detmar M, Schlapbach C, Stein J V. Intravital and whole-organ imaging reveals capture of melanoma-derived antigen by lymph node subcapsular macrophages leading to widespread deposition on follicular dendritic cells. Front Immunol. 2015;6(MAR). doi:10.3389/fimmu.2015.00114

24. Pucci F, Garris C, Lai CP, et al. SCS macrophages suppress melanoma by restricting tumor-derived vesicle-B cell interactions. Published online 2016. 
doi:10.1126/science.aaf1328

25. Louie DAP, Liao S. Lymph Node Subcapsular Sinus Macrophages as the Frontline of Lymphatic Immune Defense. Front Immunol. 2019;10(FEB):347. doi:10.3389/FIMMU.2019.00347

26. Björk Gunnarsdottir F, Auoja N, Bendahl P-O, Rydén L, Fernö M, Leandersson K. Colocalization of CD169+ macrophages and cancer cells in lymph node metastases of breast cancer patients is linked to improved prognosis and PDL1 expression. Oncoimmunology. 2020;9(1):1848067. doi:10.1080/2162402X.2020.1848067

27. Tacconi C, Commerford CD, Dieterich LC, et al. CD169+ lymph node macrophages have protective functions in mouse breast cancer metastasis. Cell Rep. 2021;35(2):108993. doi:10.1016/j.celrep.2021.108993

684

28. Balkwill F, Charles KA, Mantovani A. Smoldering and polarized inflammation in the 685 initiation and promotion of malignant disease. Cancer Cell. 2005;7(3):211-217. doi:10.1016/j.ccr.2005.02.013

29. Mantovani A, Allavena P, Sica A, Balkwill F. Cancer-related inflammation. Nature.

688 2008;454(7203):436-444. doi:10.1038/nature07205

689

30. Cassetta L, Fragkogianni S, Sims AH, Coussens LM, Smith HO, Pollard Correspondence JW. Human Tumor-Associated Macrophage and Monocyte Transcriptional Landscapes Reveal Cancer-Specific Reprogramming, Biomarkers, and Therapeutic Targets. Published online 2019. doi:10.1016/j.ccell.2019.02.009

31. Birmingham KG, O'Melia MJ, Bordy S, et al. Lymph Node Subcapsular Sinus Microenvironment-On-A-Chip Modeling Shear Flow Relevant to Lymphatic Metastasis and Immune Cell Homing. iScience. 2020;23(11):101751. doi:10.1016/J.ISCI.2020.101751

32. Balkwill F, Mantovani A. Inflammation and cancer: Back to Virchow? Lancet.

33. Spranger S, Luke JJ, Bao R, et al. Density of immunogenic antigens does not explain the presence or absence of the T-cell-inflamed tumor microenvironment in melanoma. Proc Natl Acad Sci U S A. 2016;113(48):E7759-E7768. doi:10.1073/pnas.1609376113

34. Trujillo JA, Sweis RF, Bao R, Luke JJ. T cell-inflamed versus Non-T cell-inflamed tumors: a conceptual framework for cancer immunotherapy drug development and combination therapy selection. Cancer Immunol Res. 2018;6(9):990-1000. 
heat on non-immunoreactive tumours: opportunities for clinical development. Lancet Oncol. 2020;21(9):e419-e430. doi:10.1016/S1470-2045(20)30234-5

710

711

712

713

714

715

716

717

718

719

720

721

722

723

724

725

726

727

728

729

730

731

732

733

734

735

736

737

738

739

740

741

742

36. Voronov E, Dotan S, Krelin $\mathrm{Y}$, et al. Unique versus redundant functions of IL-1 $\alpha$ and IL-1 $\beta$ in the tumor microenvironment. Front Immunol. 2013;4(JUL):177. doi:10.3389/fimmu.2013.00177

37. RN A, S D, M E, et al. The involvement of IL-1 in tumorigenesis, tumor invasiveness, metastasis and tumor-host interactions. Cancer Metastasis Rev. 2006;25(3):387-408. doi:10.1007/S10555-006-9004-4

38. Kaplanov I, Carmi Y, Kornetsky R, et al. Blocking IL-1 $\beta$ reverses the immunosuppression in mouse breast cancer and synergizes with anti-PD-1 for tumor abrogation. Proc Natl Acad Sci U S A. 2019;116(4):1361-1369. doi:10.1073/pnas.1812266115

39. Mantovani A, Dinarello CA, Molgora M, Garlanda C. Interleukin-1 and Related Cytokines in the Regulation of Inflammation and Immunity. Immunity. 2019;50(4):778795. doi:10.1016/j.immuni.2019.03.012

40. Lust JA, Lacy MQ, Zeldenrust SR, et al. Induction of a chronic disease state in patients with smoldering or indolent multiple myeloma by targeting interleukin $1 \beta$ induced interleukin 6 production and the myeloma proliferative component. Mayo Clin Proc. 2009;84(2):114-122. doi:10.4065/84.2.114

41. Hong DS, Hui D, Bruera E, et al. MABp1, a first-in-class true human antibody targeting interleukin-1 $\alpha$ in refractory cancers: An open-label, phase 1 dose-escalation and expansion study. Lancet Oncol. 2014;15(6):656-666. doi:10.1016/S14702045(14)70155-X

42. Hong DS, Janku F, Naing A, et al. Xilonix, a novel true human antibody targeting the inflammatory cytokine interleukin-1 alpha, in non-small cell lung cancer. Invest New Drugs. 2015;33(3):621-631. doi:10.1007/s10637-015-0226-6

43. Lust JA, Lacy MQ, Zeldenrust SR, et al. Reduction in C-reactive protein indicates successful targeting of the IL-1/IL-6 axis resulting in improved survival in early stage multiple myeloma. Am J Hematol. 2016;91(6):571-574. doi:10.1002/ajh.24352

44. Hickish $\mathrm{T}$, Andre $\mathrm{T}$, Wyrwicz $\mathrm{L}$, et al. MABp1 as a novel antibody treatment for advanced colorectal cancer: a randomised, double-blind, placebo-controlled, phase 3 study. Lancet Oncol. 2017;18(2):192-201. doi:10.1016/S1470-2045(17)30006-2

45. Janssen LME, Ramsay EE, Logsdon CD, Overwijk WW. The immune system in cancer metastasis: Friend or foe? J Immunother Cancer. 2017;5(1):79.

doi:10.1186/s40425-017-0283-9 
46. Garner $\mathrm{H}$, de Visser KE. Immune crosstalk in cancer progression and metastatic spread: a complex conversation. Nat Rev Immunol. 2020;20(8):483-497. doi:10.1038/s41577-019-0271-z

47. Hiam-Galvez KJ, Allen BM, Spitzer MH. Systemic immunity in cancer. Nat Rev Cancer. Published online April 9, 2021:1-15. doi:10.1038/s41568-021-00347-z

48. Edwards SC, Hoevenaar WHM, Coffelt SB. Emerging immunotherapies for metastasis. Br J Cancer. 2021;124(1):37-48. doi:10.1038/s41416-020-01160-5

49. Ralli M, Botticelli A, Visconti IC, et al. Immunotherapy in the Treatment of Metastatic Melanoma: Current Knowledge and Future Directions. J Immunol Res. 2020;2020. doi:10.1155/2020/9235638

50. Michielin O, Atkins MB, Koon HB, Dummer R, Ascierto PA. Evolving impact of longTerm survival results on metastatic melanoma treatment. $J$ Immunother Cancer. 2020;8(2):948. doi:10.1136/jitc-2020-000948

51. Lee $\mathrm{C}$ kun, Jeong $\mathrm{S}$ hwan, Jang $\mathrm{C}$, et al. Tumor metastasis to lymph nodes requires YAP-dependent metabolic adaptation. Science (80- ). 2019;363(6427):644-649. doi:10.1126/science.aav0173

52. Pizzagalli DU, Latino I, Pulfer A, et al. Characterization of the Dynamic Behavior of Neutrophils Following Influenza Vaccination. Front Immunol. 2019;10. doi:10.3389/fimmu.2019.02621

53. Fu X-Q, Liu B, Wang Y-P, et al. Activation of STAT3 is a key event in TLR4 signalingmediated melanoma progression. Cell Death Dis 2020 114. 2020;11(4):1-15. doi:10.1038/s41419-020-2440-1

54. A S, R S, O E, et al. STAT3 promotes melanoma metastasis by CEBP-induced repression of the MITF pathway. Oncogene. 2021;40(6):1091-1105. doi:10.1038/S41388-020-01584-6

55. Malvehy J, Puig S. Expression of Stat3 and Src proteins in mucosal, cutaneous, and metastatic melanoma. J Am Acad Dermatol. 2005;52(3):P144. doi:10.1016/J.JAAD.2004.10.584

56. Lin W-H, Chang Y-W, Hong M-X, et al. STAT3 phosphorylation at Ser727 and Tyr705 differentially regulates the EMT-MET switch and cancer metastasis. Oncogene 2020 404. 2020;40(4):791-805. doi:10.1038/s41388-020-01566-8

57. Zerdes I, Wallerius M, Sifakis EG, et al. STAT3 Activity Promotes Programmed-Death Ligand 1 Expression and Suppresses Immune Responses in Breast Cancer. Cancers (Basel). 2019;11(10). doi:10.3390/CANCERS11101479

58. Zou S, Tong Q, Liu B, Huang W, Tian Y, Fu X. Targeting STAT3 in Cancer 
Immunotherapy. Mol Cancer 2020 191. 2020;19(1):1-19. doi:10.1186/S12943-02001258-7

59. Hoeller $\mathrm{C}$. The future of combination therapies in advanced melanoma. memo - Mag Eur Med Oncol 2020 133. 2020;13(3):309-313. doi:10.1007/S12254-020-00640-X

60. Yu C, Liu X, Yang J, et al. Combination of Immunotherapy With Targeted Therapy: Theory and Practice in Metastatic Melanoma. Front Immunol. 2019;0(MAY):990. doi:10.3389/FIMMU.2019.00990

61. Berraondo P, Sanmamed MF, Ochoa MC, et al. Cytokines in clinical cancer immunotherapy. Br J Cancer 2018 1201. 2018;120(1):6-15. doi:10.1038/s41416-0180328-y

62. Johnson DE, O'Keefe RA, Grandis JR. Targeting the IL-6/JAK/STAT3 signalling axis in cancer. Nat Rev Clin Oncol 2018 154. 2018;15(4):234-248. doi:10.1038/nrclinonc.2018.8

63. Lippitz BE, Harris RA. Cytokine patterns in cancer patients: A review of the correlation between interleukin 6 and prognosis. Oncoimmunology. 2016;5(5). doi:10.1080/2162402X.2015.1093722

64. Kurzrock R, Hickish T, Wyrwicz L, et al. Interleukin-1 receptor antagonist levels predict favorable outcome after bermekimab, a first-in-class true human interleukin-1a antibody, in a phase III randomized study of advanced colorectal cancer. Oncoimmunology. 2019;8(3). doi:10.1080/2162402X.2018.1551651

65. Kauffmann-Guerrero D, Kahnert K, Kiefl R, et al. Systemic inflammation and pro799 inflammatory cytokine profile predict response to checkpoint inhibitor treatment in NSCLC: a prospective study. Sci Reports 2021 111. 2021;11(1):1-10. doi:10.1038/s41598-021-90397-y

66. Wang M, Zhai X, Li J, et al. The Role of Cytokines in Predicting the Response and

67. Perrichet A, Ghiringhelli F, Rébé $C$. Understanding Inflammasomes and PD-1/PD-L1 Crosstalk to Improve Cancer Treatment Efficiency. Cancers (Basel). 2020;12(12):119. doi:10.3390/CANCERS 12123550

68. Ashizawa $\mathrm{T}$, lizuka A, Maeda $\mathrm{C}$, et al. Impact of combination therapy with anti-PD-1 blockade and a STAT3 inhibitor on the tumor-infiltrating lymphocyte status. Immunol Lett. 2019;216:43-50. doi:10.1016/J.IMLET.2019.10.003 primary tumor and corresponding sentinel lymph node. Mod Pathol. 2012;25(7):1000- 
814 70. Chitty JL, Filipe EC, Lucas MC, Herrmann D, Cox TR, Timpson P. Recent advances in understanding the complexities of metastasis. F1000Research. 2018;7. doi:10.12688/F1000RESEARCH.15064.2

71. Zhou H, Lei P, Padera TP. Progression of Metastasis through Lymphatic System. Cells 2021, Vol 10, Page 627. 2021;10(3):627. doi:10.3390/CELLS10030627

72. $\mathrm{Hu}$ J, Xu J, Li M, et al. Targeting Lymph Node Sinus Macrophages to Inhibit Lymph Node Metastasis. Mol Ther Nucleic Acid. 2019;16:650-662. doi:10.1016/j.omtn.2019.04.016

73. Mantovani A, Marchesi F, Jaillon S, Garlanda C, Allavena P. Tumor-associated myeloid cells: diversity and therapeutic targeting. Cell Mol Immunol. 2021;18(3):566578. doi:10.1038/s41423-020-00613-4

74. Tacconi C, Commerford CD, Dieterich LC, et al. CD169+ lymph node macrophages have protective functions in mouse breast cancer metastasis. Cell Rep. 2021;35(2):108993. doi:10.1016/j.celrep.2021.108993

75. Cassetta L, Pollard JW. Repolarizing macrophages improves breast cancer therapy. Cell Res 2017 278. 2017;27(8):963-964. doi:10.1038/cr.2017.63

76. Crecente-Campo J, Virgilio T, Morone D, et al. Design of polymeric nanocapsules to improve their lympho-targeting capacity. Nanomedicine. 2019;14(23). doi:10.2217/nnm-2019-0206

77. Schudel A, Francis DM, Thomas SN. Material design for lymph node drug delivery. Nat Rev Mater. 2019;4(6):415. doi:10.1038/S41578-019-0110-7

78. Grabowska J, Lopez-Venegas MA, Affandi AJ, den Haan JMM. CD169+ Macrophages Capture and Dendritic Cells Instruct: The Interplay of the Gatekeeper and the General of the Immune System. Front Immunol. 2018;9(OCT):2472. doi:10.3389/FIMMU.2018.02472

79. Rider P, Kaplanov I, Romzova M, et al. The transcription of the alarmin cytokine interleukin- 1 alpha is controlled by hypoxia inducible factors 1 and 2 alpha in hypoxic cells. Front Immunol. 2012;3(SEP). doi:10.3389/FIMMU.2012.00290

80. Kurzrock R, Hickish T, Wyrwicz L, et al. Interleukin-1 receptor antagonist levels predict favorable outcome after bermekimab, a first-in-class true human interleukin-1a antibody, in a phase III randomized study of advanced colorectal cancer. Oncoimmunology. 2019;8(3). doi:10.1080/2162402X.2018.1551651

81. Horai R, Asano M, Sudo K, et al. Production of mice deficient in genes for interleukin (IL)-1 $\alpha, I L-1 \beta, I L-1 \alpha / \beta$, and IL-1 receptor antagonist shows that IL- $1 \beta$ is crucial in 
turpentine-induced fever development and glucocorticoid secretion. J Exp Med. 1998;187(9):1463-1475. doi:10.1084/jem.187.9.1463

850

82. Glaccum MB, Stocking KL, Charrier K, et al. Phenotypic and functional characterization of mice that lack the type I receptor for IL-1. J Immunol. 1997;159(7):3364-3371. http://www.ncbi.nIm.nih.gov/pubmed/9317135

83. Jung S, Aliberti J, Graemmel P, et al. Analysis of Fractalkine Receptor CX3CR1 Function by Targeted Deletion and Green Fluorescent Protein Reporter Gene Insertion. Mol Cell Biol. 2000;20(11):4106-4114. doi:10.1128/mcb.20.11.41064114.2000

84. Zheng GXY, Terry JM, Belgrader $P$, et al. Massively parallel digital transcriptional profiling of single cells. Nat Commun. 2017;8(1):1-12. doi:10.1038/ncomms14049

85. Butler A, Hoffman P, Smibert P, Papalexi E, Satija R. Integrating single-cell transcriptomic data across different conditions, technologies, and species. Nat Biotechnol. 2018;36(5):411-420. doi:10.1038/nbt.4096

86. Stuart T, Butler A, Hoffman P, et al. Comprehensive Integration of Single-Cell Data. Cell. 2019;177(7):1888-1902.e21. doi:10.1016/j.cell.2019.05.031

87. Catlin PA, Chartrand G. Introductory Graph Theory. Am Math Mon. 1987;94(5):483. doi:10.2307/2322751

88. Meyer B. Self-Organizing Graphs-A Neural Network Perspective of Graph Layout.

89. Kanehisa M, Furumichi M, Tanabe M, Sato Y, Morishima K. KEGG: New perspectives on genomes, pathways, diseases and drugs. Nucleic Acids Res. 2017;45(D1):D353D361. doi:10.1093/nar/gkw1092

90. Szklarczyk D, Gable AL, Lyon D, et al. STRING v11: Protein-protein association networks with increased coverage, supporting functional discovery in genome-wide experimental datasets. Nucleic Acids Res. 2019;47(D1):D607-D613. doi:10.1093/nar/gky1131

91. Mao C, Xiao W. A comprehensive algorithm for evaluating node influences in social networks based on preference analysis and random walk. Complexity. 2018;2018. doi:10.1155/2018/1528341

92. Bihari A, Pandia MK. Eigenvector centrality and its application in research professionals' relationship network. In: 2015 1st International Conference on Futuristic Trends in Computational Analysis and Knowledge Management, ABLAZE 2015. Institute of Electrical and Electronics Engineers Inc.; 2015:510-514. doi:10.1109/ABLAZE.2015.7154915 
883

884

885

886

887

888

889

890

891

892

893

894

895

896

897

898

899

900

901

902

903

904

905

in Computer Science. Vol 3404. Springer Verlag; 2005:533-544. doi:10.1007/978-3-

$$
\text { 540-31856-9_44 }
$$

94. Newman MEJ. A measure of betweenness centrality based on random walks. Soc Networks. 2003;27(1):39-54. Accessed April 9, 2021. http://arxiv.org/abs/condmat/0309045

95. Mones E. Hierarchy in directed random networks. Phys Rev E - Stat Nonlinear, Soft Matter Phys. 2013;87(2):022817. doi:10.1103/PhysRevE.87.022817

96. C T, E G, AJ A, et al. PQR309 Is a Novel Dual PI3K/mTOR Inhibitor with Preclinical Antitumor Activity in Lymphomas as a Single Agent and in Combination Therapy. Clin Cancer Res. 2018;24(1):120-129. doi:10.1158/1078-0432.CCR-17-1041

97. TC C. Drug combination studies and their synergy quantification using the ChouTalalay method. Cancer Res. 2010;70(2):440-446. doi:10.1158/0008-5472.CAN-091947

98. Gaudio E, Tarantelli C, Spriano F, et al. Targeting CD205 with the antibody drug conjugate MEN1309/OBT076 is an active new therapeutic strategy in lymphoma models. Haematologica. 2020;105(11):2584-2591. doi:10.3324/HAEMATOL.2019.227215

99. Meyer CT, Wooten DJ, Paudel BB, et al. Quantifying Drug Combination Synergy along Potency and Efficacy Axes. Cell Syst. 2019;8(2):97-108.e16. doi:10.1016/J.CELS.2019.01.003

100. Pfaffl MW. A new mathematical model for relative quantification in real-time RT-PCR. Nucleic Acids Res. 2001;29(9). doi:10.1093/nar/29.9.e45 
908 Figure 1. Mouse melanoma metastases growth in the sentinel LN. (A) FACS plot showing 909 fluorescent expression of the mCherry transduced B16-F1 melanoma cells. (B) Schematic 910 representation of the tumor model, including primary tumor engraftment (left) and migration of cells to

911 the $\operatorname{SLN}$ (right). (C) Representative images and (D) quantification of IVIS time-course showing 912 increasing primary tumor fluorescence (red). (E, left) Primary tumor (red arrow), draining sentinel LN 913 (white circle) and (E, right) comparison between metastatic and healthy $L N$ at week three p.t.i.. (F) Time914 course of metastatic cell invasion of the sLN quantified by FACS. (G) Confocal micrograph of $S L N$ at 915 week one, two and three p.t.i., showing the position of B16 melanoma (red) with respect to CD21/35 916 (blue) follicular dendritic cells and CX3CR1 $1^{+}$(green) myeloid cells. SS, IF, F, T and M stand for 917 subcapsular sinus, inter-follicular, follicular, $T$ cells and medullary areas, respectively. (H) Quantification 918 of total metastatic area in the $S L N$, measured by confocal microscopy. (I) Quantification of tumor cells 919 in the different compartments of the $L N$ at week two and three p.t.i.. (J) Metastatic ratio, defined as the 920 number of mice with metastases in the target organ divided by the total number of implanted mice, at 921 week three p.t.i. iLN stands for inguinal $L N$.

922

Figure 2. Pro-tumoral release of IL-1a in the metastatic $L N$

(A) Progressive increase in the size of the $S L N$ correlated with the increase in the total number of immune cells, measured by flow cytometry. (B) Quantification of inflammatory cytokines in the supernatant of metastatic (red) and non-metastatic (white) LN. (C) Time course kinetics showing IL-1a release in the $s L N$ during the first three weeks p.t.i.. (D) Quantification of IL-1 $\alpha$ in the $s L N$ at three weeks p.t.i. of different cancer models, including breast cancer (E0771) and the melanoma B16-F10. (E) Flow cytometric quantification of $L N$ metastatic cells in animals treated with IL-1a depleting antibody or recombinant IL-1 $\alpha$ in comparison to B16-F1 untreated group and PBS injected. (F) LN metastatic ratio in mice untreated or treated with anti-IL-1 $\alpha$ antibody or recombinant IL-1 $\alpha$ at week three p.t.i.. (G) Metastatic cells in the $s L N$ of wild type and IL-1a KO mice three weeks p.t.i..

934 Figure 3. SSM are the main source of IL-1a. (A) UMAP plot of cell populations, identified by scSeq, in the metastasized sLN three weeks p.t.i.. (B) Percentage of cells expressing il1a and (C) average il1a expression in the cells of the $s L N$ three weeks p.ti.., measured by scSeq. (D) IL-1a quantification in metastasized SLN supernatant of mice depleted for macrophages by clodronate liposome (CLL) injection in comparison to untreated metastasized and non-metastasized LN. (E) Flow cytometric histograms showing presence, three weeks p.t.i., of IL-1 $\alpha^{+}$(red) and IL-10 negative (gray) SSM. (F)

940 Flow cytometric quantification of the number of $I L-1 \alpha^{+}$cells among the three major subtypes of 941 macrophages in the sLN three weeks p.t.i. in comparison to negative controls. SSM, MCM and MM 942 stand for Subcapsular Sinus Macrophages (CD169+ F4/80), Medullary Cord Macrophages (CD169$\left.943 \mathrm{~F} 4 / 8 \mathrm{O}^{+}\right)$and Medullary Macrophages $\left(\mathrm{CD} 169^{+} \mathrm{F} 4 / 80^{+}\right)$, respectively. (G) Confocal micrograph showing 944 the whole $S L N$ (left) and magnifications of the metastatic region (center and right) indicating IL-1a and 


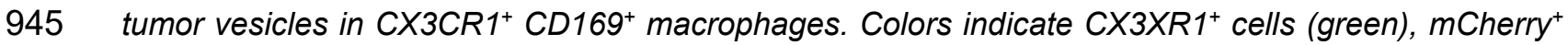
946 melanoma (red), CD169+ macrophages (cyan) and IL-1a (white). (H) Flow cytometric quantification 947 indicating the number of each subtype of tumor ${ }^{+}$macrophages. (I) Confocal representative images of

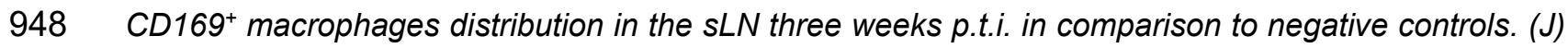
949 Quantification of CD169 fluorescence in the main regions of the $L N$ three weeks p.t.i., indicating 950 disruption of CD169 layer (white arrows) in the SS overlying the metastatic area (SSMET).

951

952 Figure 4. Direct effect of pro-tumoral IL-1a on metastatic cells. (A) Flow cytometry quantification of 953 mean fluorescence intensity (MFI) indicating IL-1R1 expression in the $S L N$ cell populations. (B) Flow 954 cytometric quantification of metastatic cells and (C) metastatic ratio in wild type and IL-1R1 knock-out 955 mice, three weeks p.t.i. (D) Proliferation assay (MTT) of B16-F1 treated with recombinant IL-1a for 24 $956 h$ in comparison to untreated cells. (E) Proliferation assay (MTT) of human melanoma A375 treated with 957 human recombinant $I L-1 \alpha$ for $72 h$, in comparison to untreated cells. (F) qPCR quantification of the Myd88 gene in B16-F1 stimulated with recombinant IL-1a in comparison to unstimulated.

959

960 Figure 5. IL-1a induces STAT3 expression and phosphorylation in tumor. (A) STRING graph

961 representing the most influential genes obtained by node influence analysis of il1a enriched pathways

962 in scSeq data of the metastatic area of the $s L N$. The influence of each node is expressed in a

963 colorimetric scale. (B) Bar plot showing node influence of the ten most influential genes in il1a

964 pathways three weeks p.t.i.. STAT3 is highlighted (red). (C) Bar plot indicating differential expression

965 (DE) of the ten most influential nodes in tumor following IL-1 $\alpha$ block in comparison to untreated mice.

966 STAT3 is highlighted (red). (D) Average STAT3 expression in each cell population of the metastatic

$967 s L N$. (E) qPCR quantification of STAT3 expression in B16-F1 following recombinant IL-1a

968 administration. Quantification of (F) STAT3 and (G) pSTAT3, measured by immunoblot, in B16-F1

969 after IL-1 $\alpha$ treatment. Immunoblot quantifications of (H) STAT3 and (I) pSTAT3 in human melanoma

970 A375 following exposure to IL-1 $\alpha$. (J) Flow cytometric quantification of metastatic cells in mouse sLN

971 of mice treated with anti-IL-1 $\alpha$ antibody, the STAT3 inhibitor stattic or their combination, in comparison

972 to untreated. (K) Proliferation of mouse melanoma upon combination therapy with anti-IL-1a stattic at

973 different concentrations, measured by MTT assay. 
A

B16-F1 Fluorescence Transduction

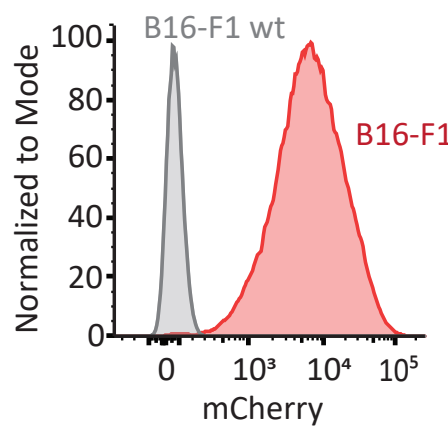

B

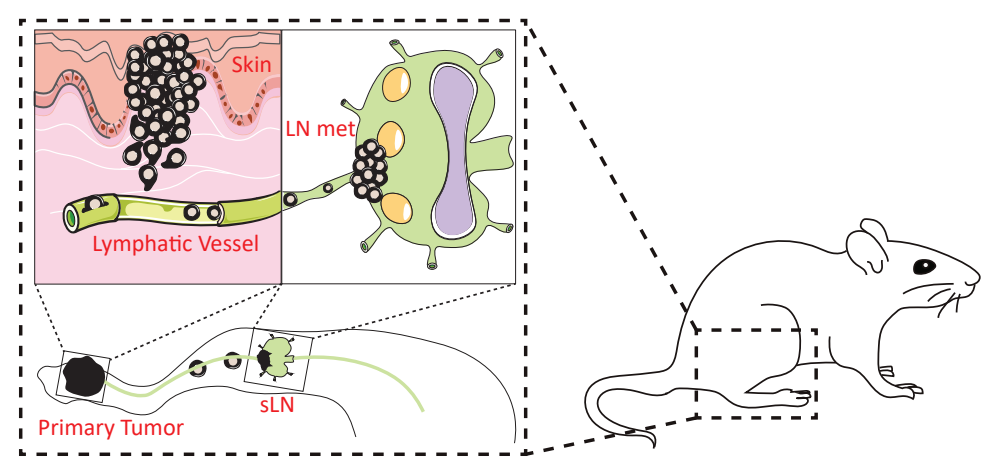

C

Fluorescence Intensity

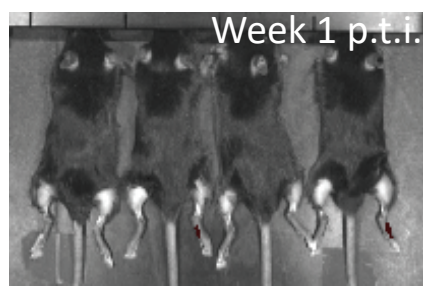

D

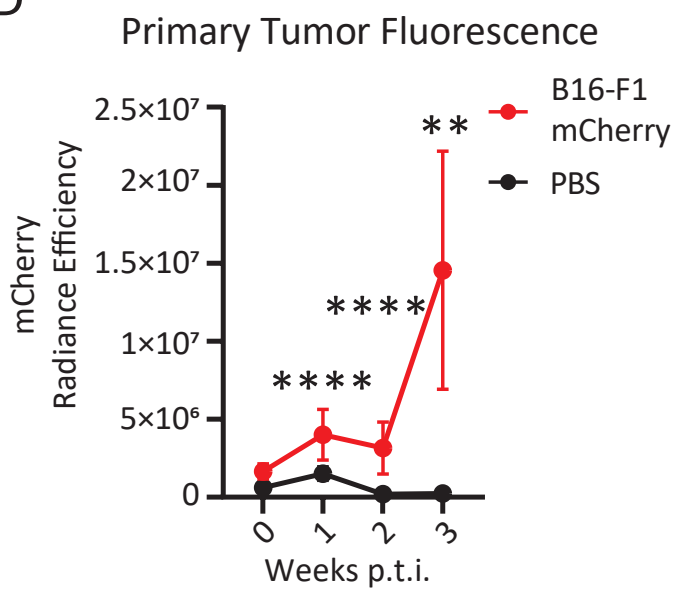

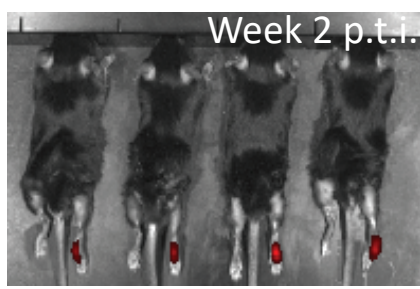

E

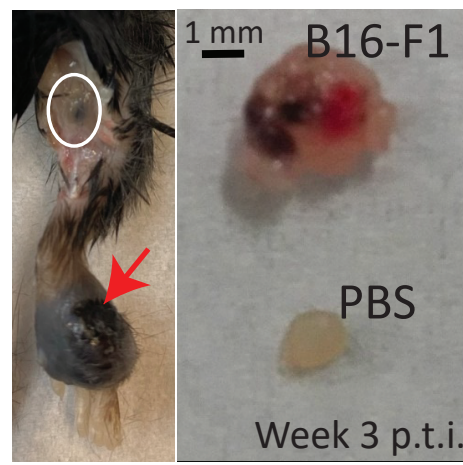

$\mathrm{F}$

Metastatic Cells in the sLN

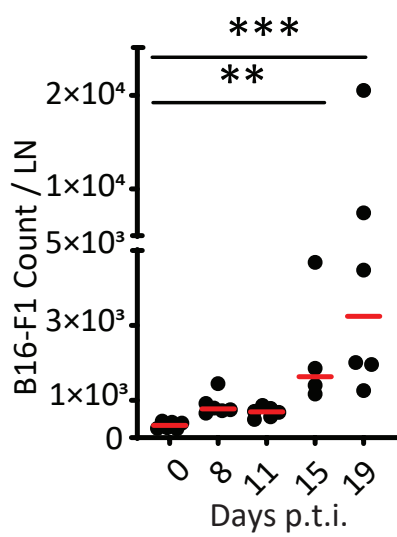

G
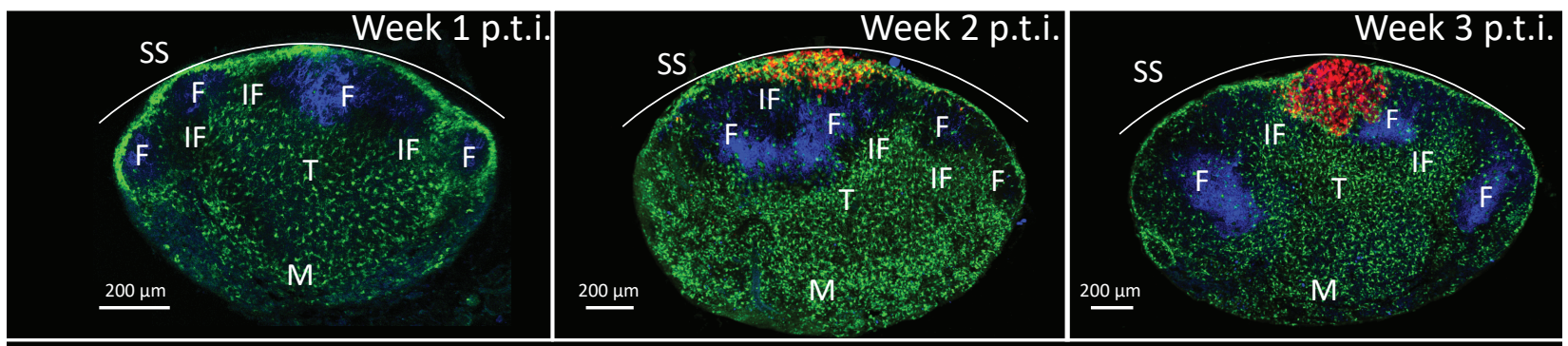

CX3CR1 B16-F1 CD21/35

$\mathrm{H}$

Metastatic Area

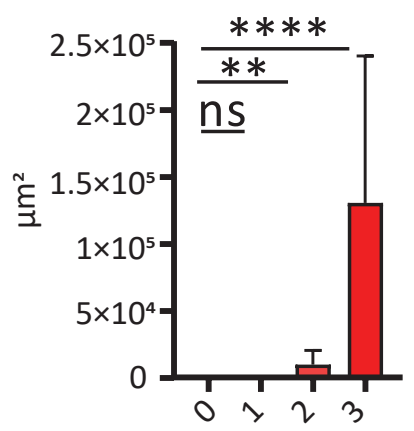

Weeks p.t.i.
Proportion of Metastasic Cells Propagating in the sLN Regions
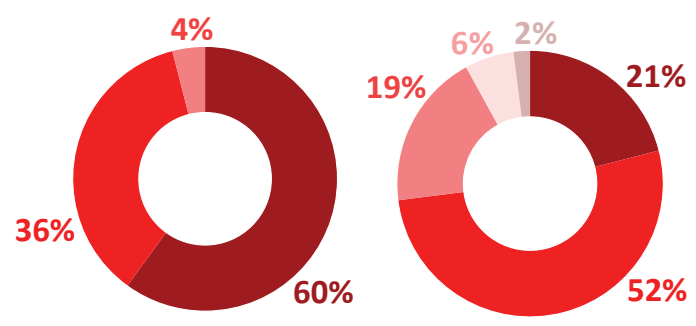

Week 3 p.t.i.
J

Metastasic Ratio

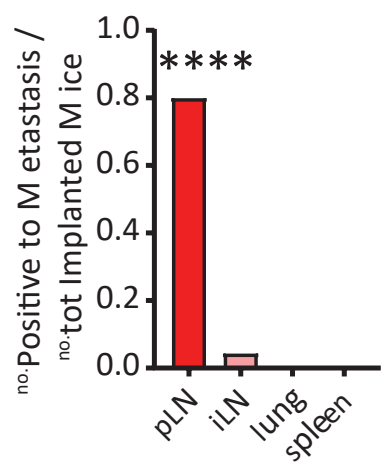

Target Organ 
Figure 2. Pro-tumoral release of IL-1 $\alpha$ in the metastatic LN

A

Total Immune Cells

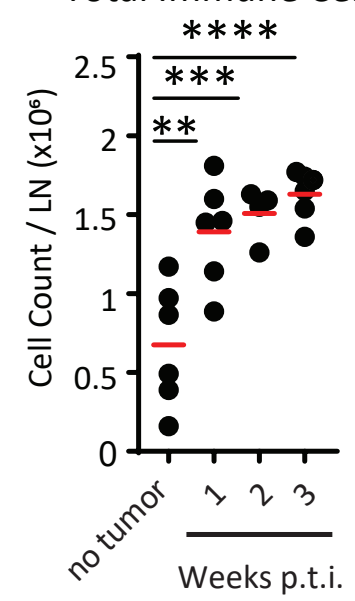

D

Tumor Model

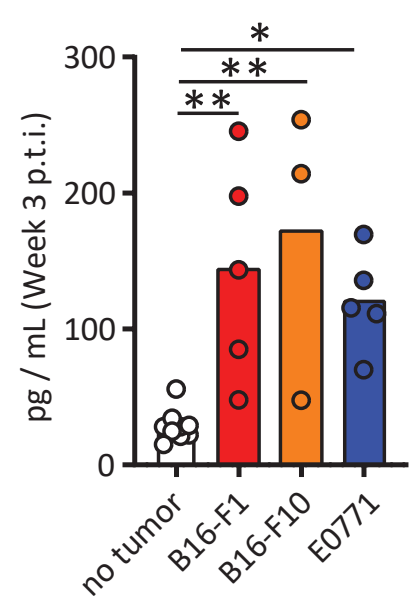

B

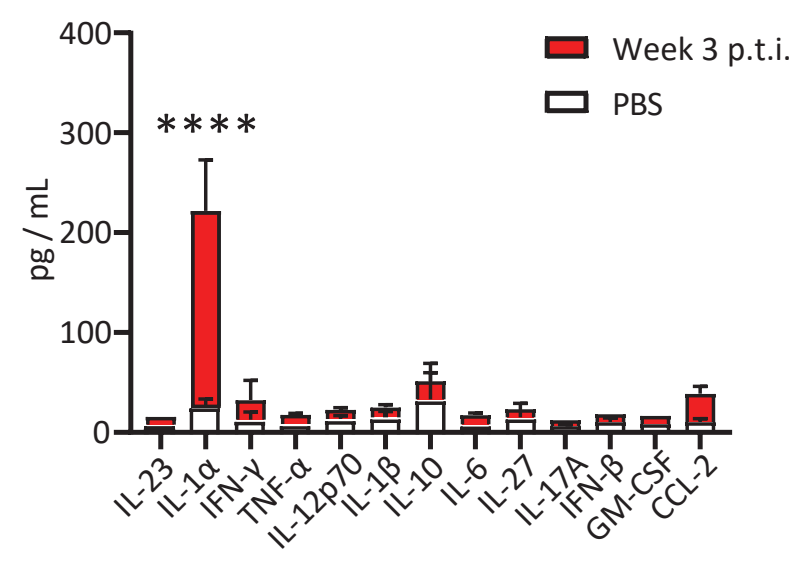

$E$

Metastatic Cells

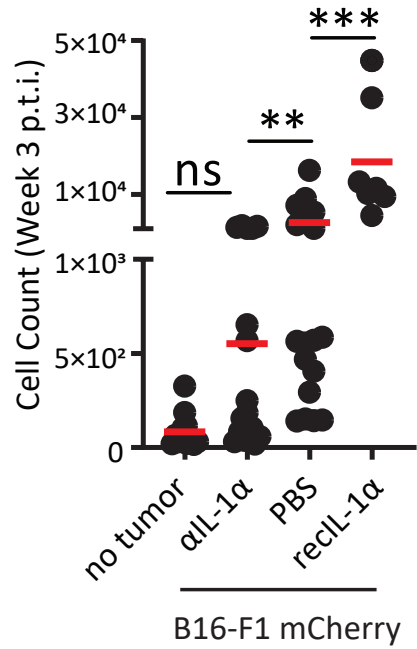

F

Metastasic Ratio

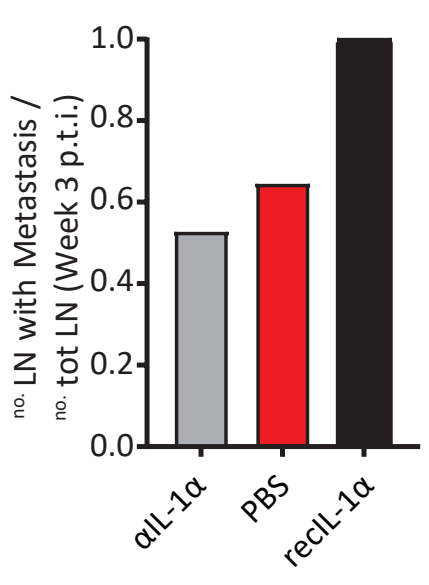

Treatment
C

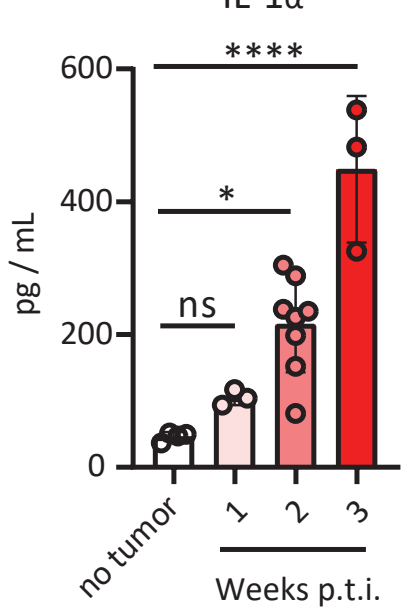

G

Metastatic Cells

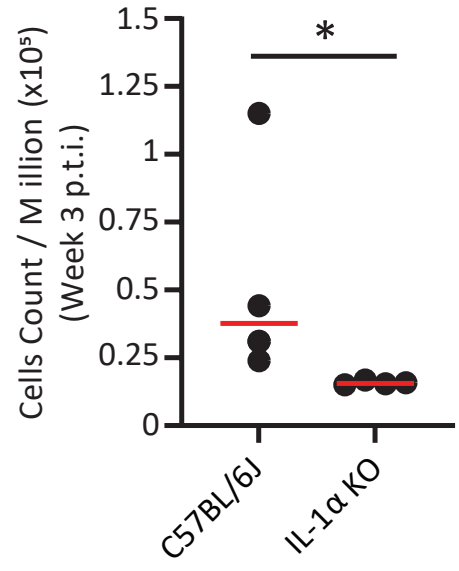

Mouse Strain 


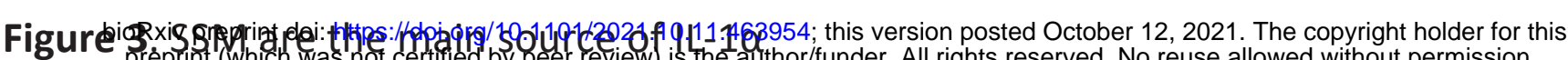
preprint (Which was not certmed by peer review) is the author/funder. All rights reserved. No reuse allowed without permission.

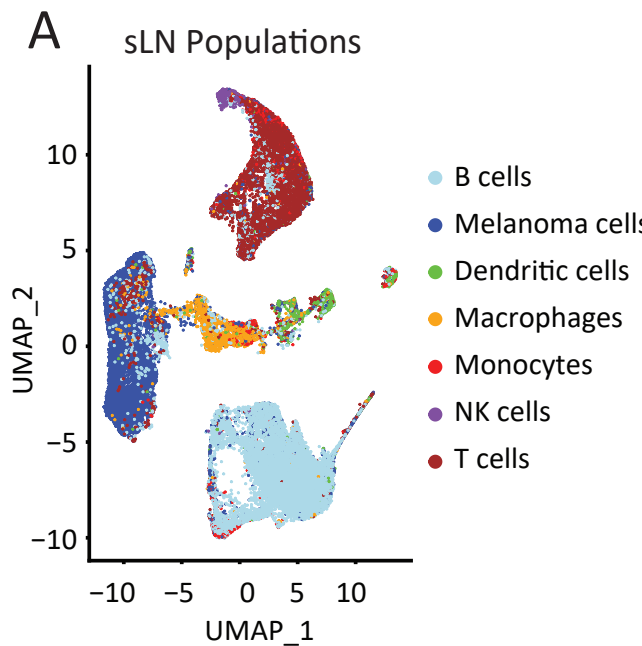

D

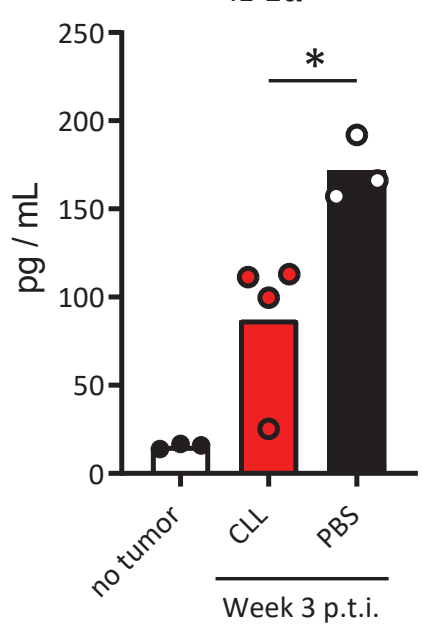

B
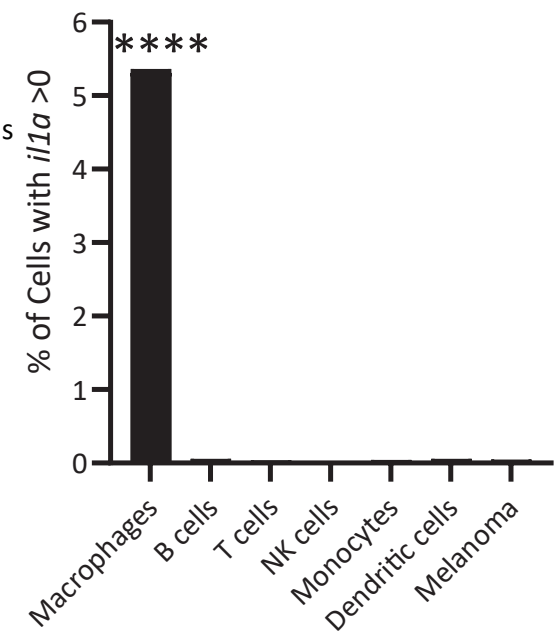

E

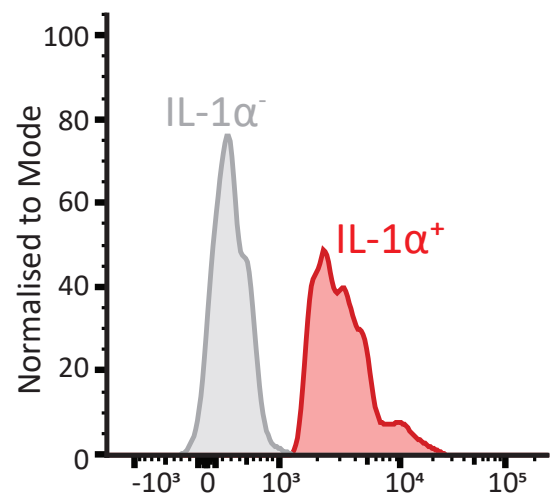

IL-1 $\alpha$ Fluorescence Intensity

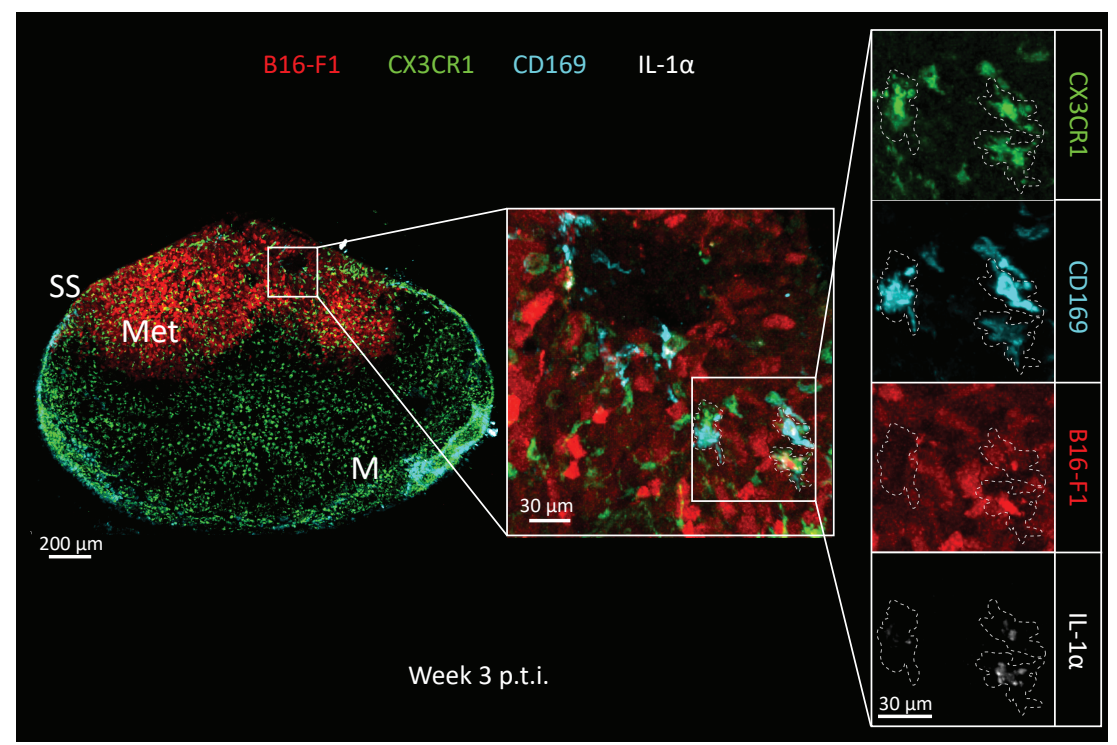

$\mathrm{H}$

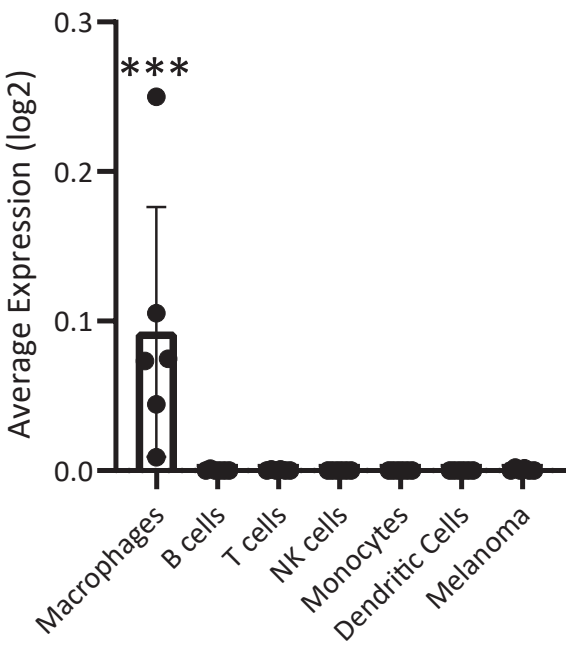

F

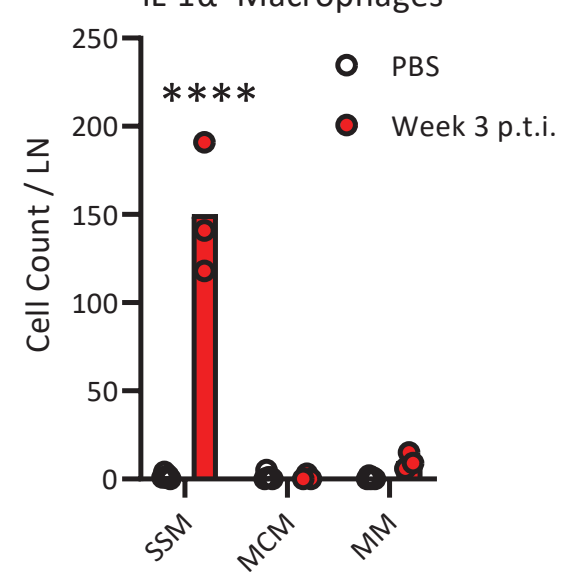

Macrophages Subtypes

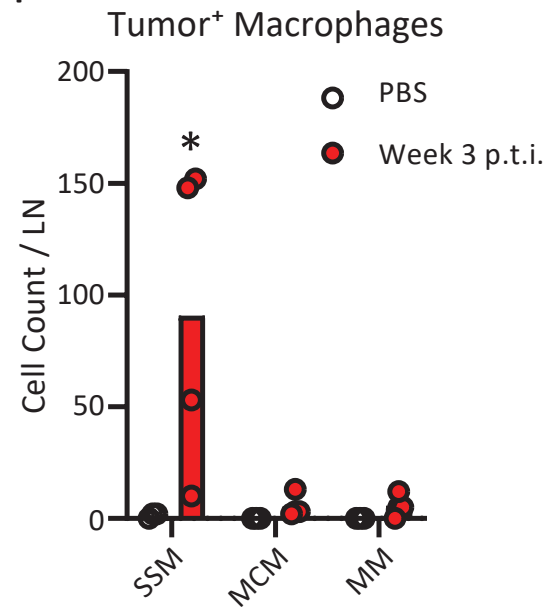

Macrophages Subtypes

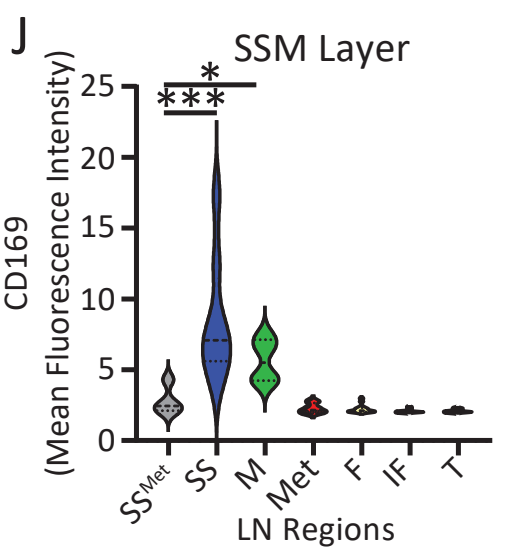


Figure 4. SSM derived IL-1 $\alpha$ directly supports tumor proliferation

A

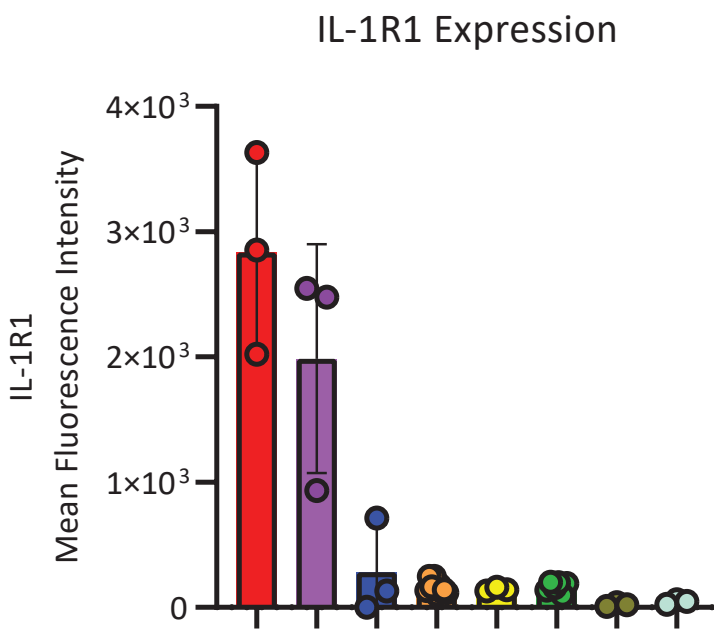

$\mathrm{D}$

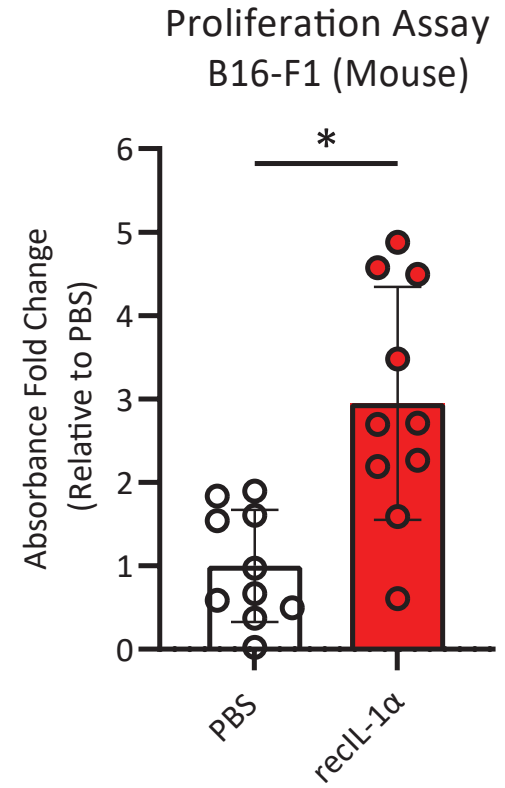

B

Metastatic Cells
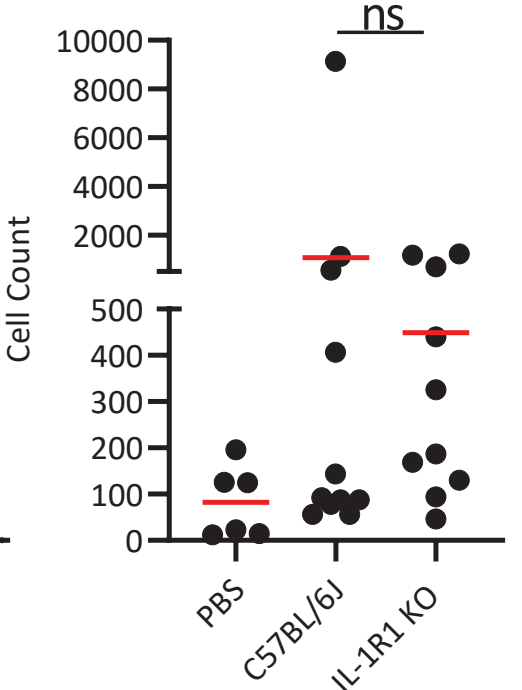

$E$

Proliferation Assay A3775 (Human)

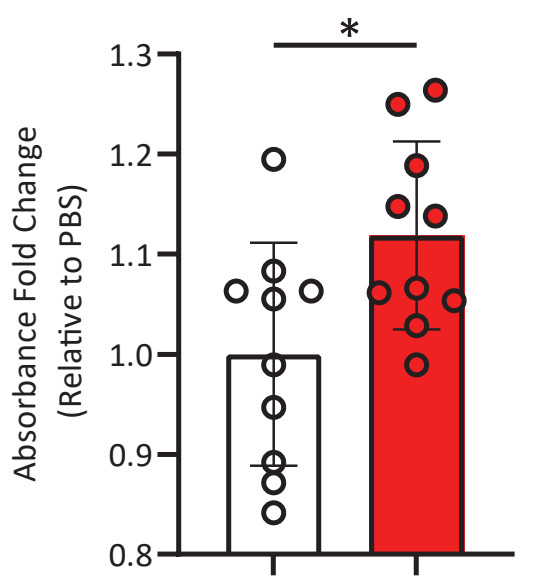

$\beta^{s}$
C

Metastasic Ratio

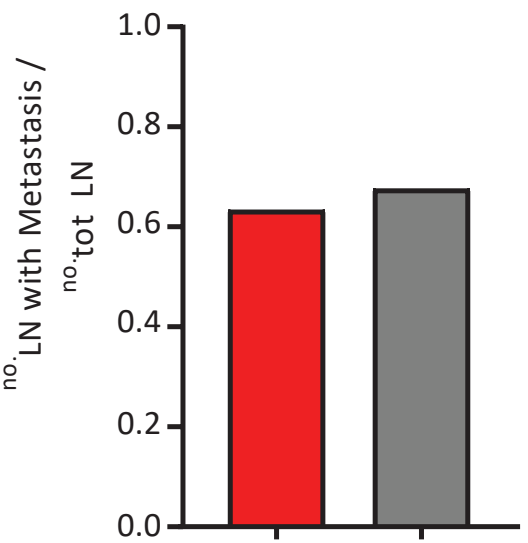

(6)

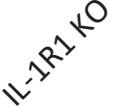

F

MYD88

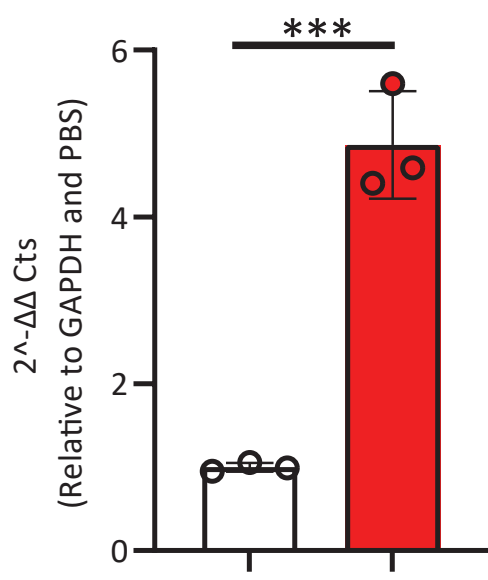

$\beta^{s}$

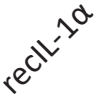




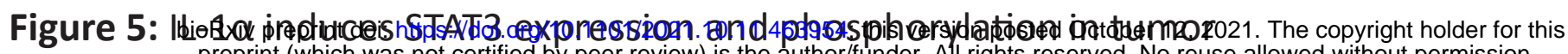

A preprint (which was not certified by peer review) is the author/funder. All rights reserved. No re
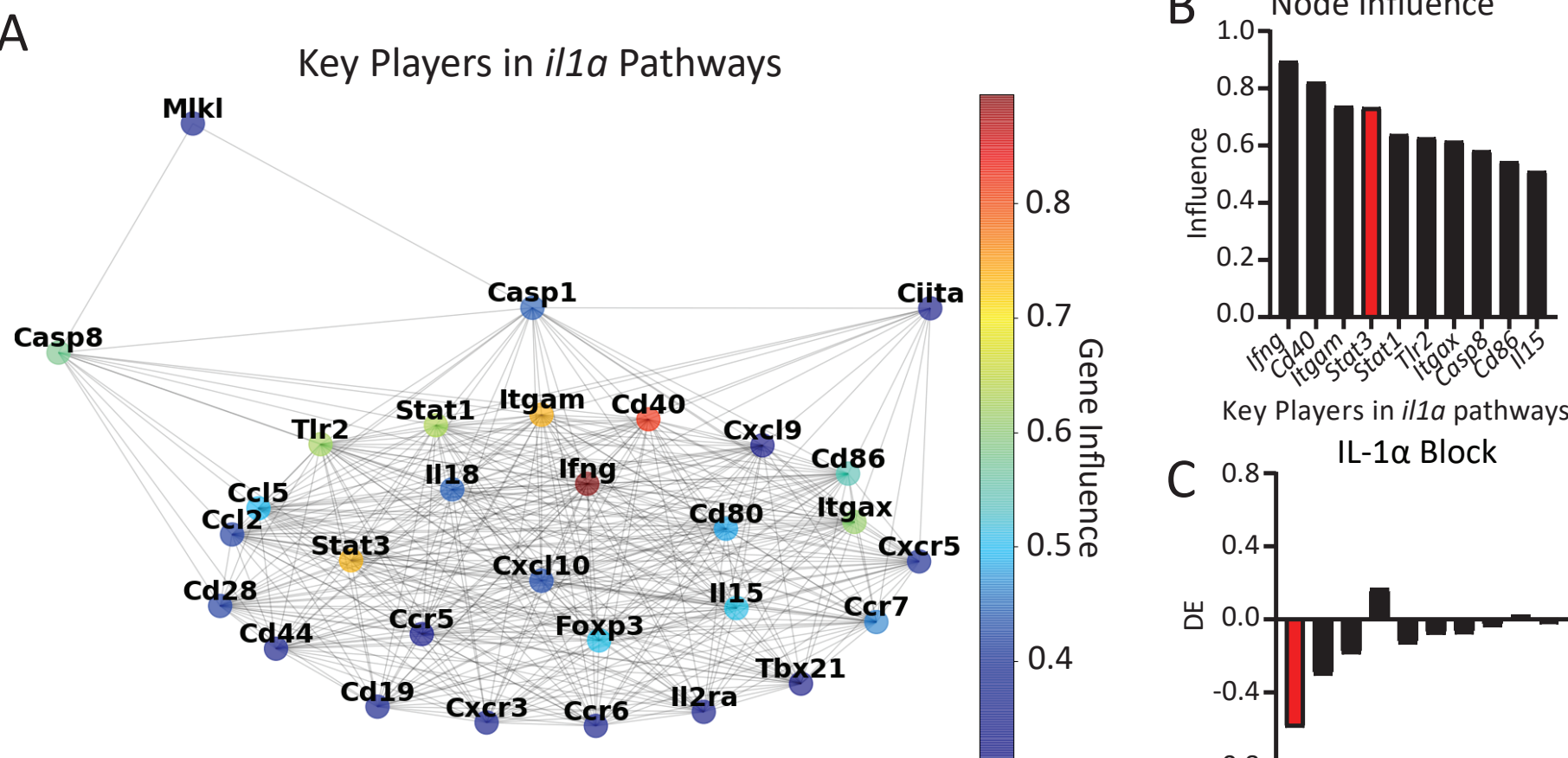

0.8

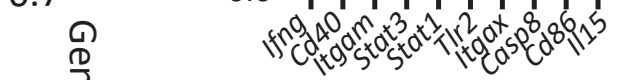

$0.6 \frac{\frac{D}{D}}{\frac{D}{3}}$

0.4

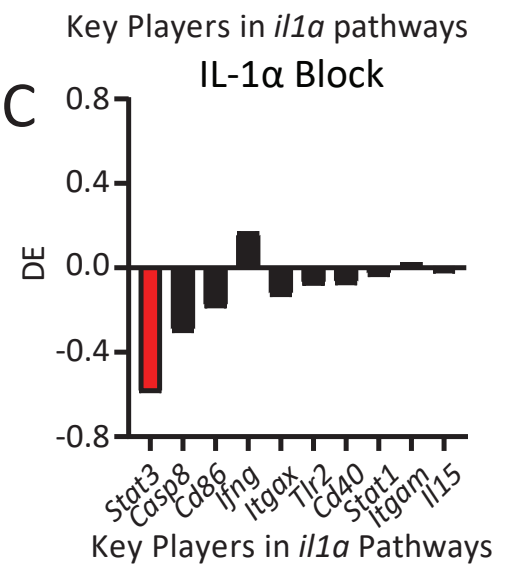

D

STAT3 Expression

E STAT3 Expression

$\mathrm{F}$

STAT3 $\quad G$

pSTAT3

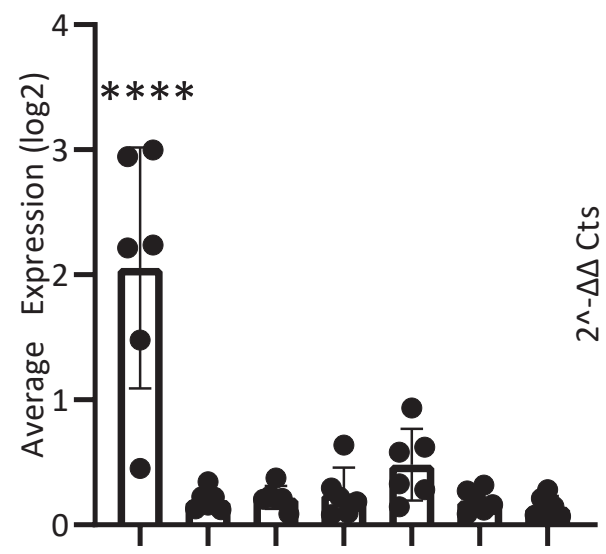

$\mathrm{H}$

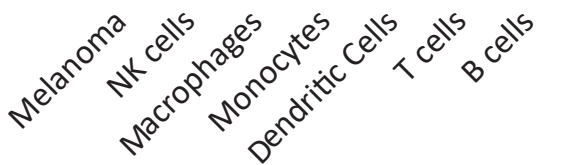

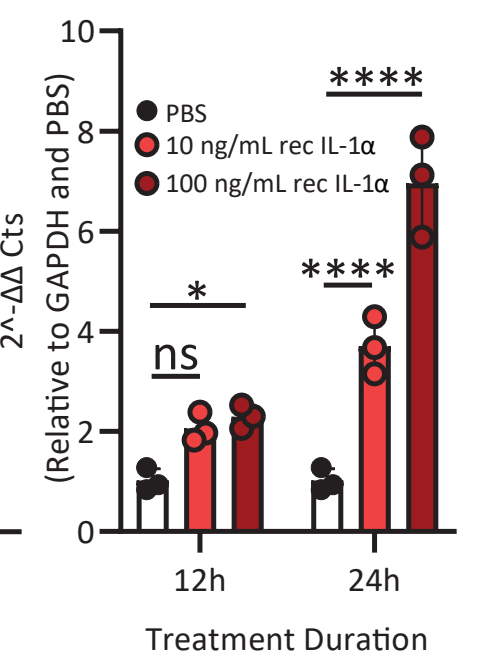

Treatment Duration
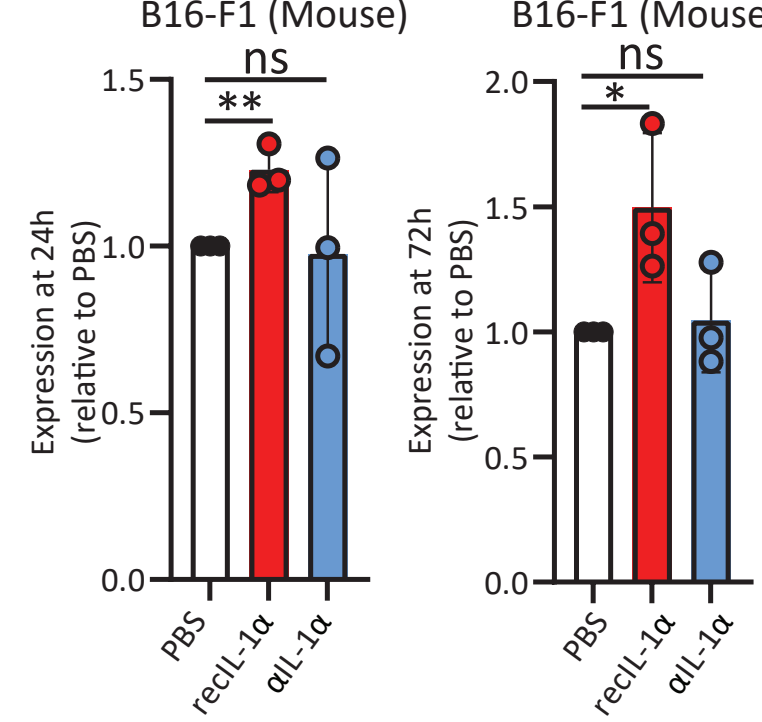

STAT3

A375 (Human)

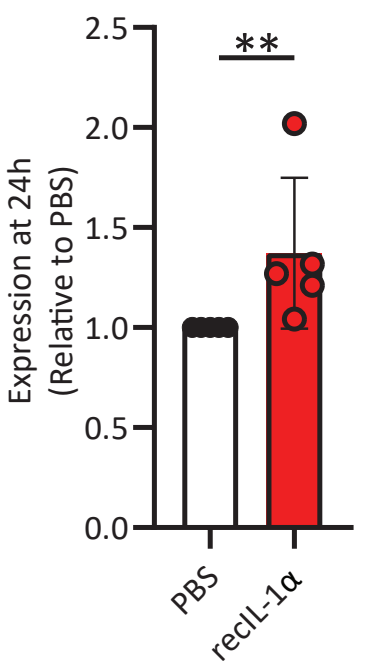

pSTAT3

A375 (Human)

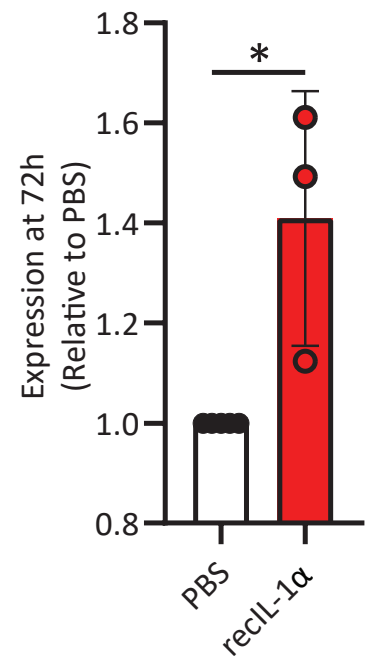

J

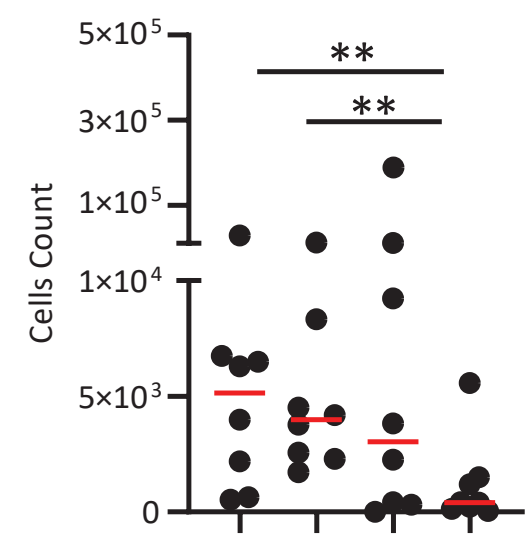

Metastatic Cells
K

Combination Therapy $\alpha \mathrm{IL}-1 \alpha+$ stattic

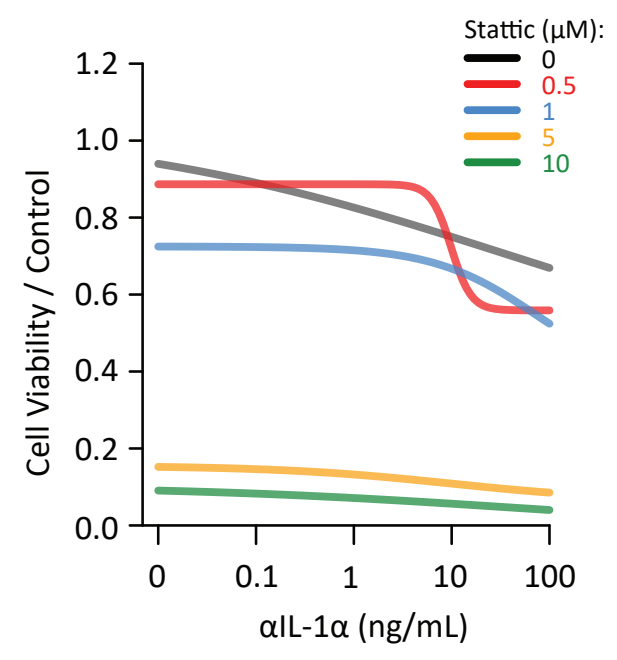

